\title{
Accelerating Kohn-Sham Response Theory using Density fitting and the Auxiliary-Density-Matrix Method
}

\author{
Chandan Kumar $^{1}$ | Heike Fliegl ${ }^{1}$ | Frank Jensen ${ }^{2}$ | \\ Andrew M. Teale ${ }^{1,3,4}$ | Simen Reine ${ }^{1}$ | Thomas \\ Kjærgaard $^{5}$
}

\begin{abstract}
${ }^{1}$ Hylleraas Centre for Quantum Molecular Sciences, Department of Chemistry, University of Oslo, P.O.Box 1033, N-1315 Blindern, Norway

${ }^{2}$ Department of Chemistry, Aarhus University, Langelandsgade 140, DK-8000 Aarhus C, Denmark

${ }^{3}$ School of Chemistry, University of Nottingham, University Park, Nottingham NG7 2RD, United Kingdom

${ }^{4}$ Centre for Advanced Study at the Norwegian Academy of Science and Letters. Drammensveien 78, N-0271 Oslo, Norway ${ }^{5}$ qLEAP Center for Theoretical Chemistry, Department of Chemistry, Aarhus University, Langelandsgade 140, DK-8000 Aarhus C, Denmark
\end{abstract}

\section{Correspondence \\ Chandan Kumar and Simen Reine, Hylleraas Centre for Quantum Molecular Sciences, Department of Chemistry, University of Oslo, P.O.Box 1033, N-1315, Blindern, Norway. \\ Email: chandan.kumar@kjemi.uio.no simen.reine@kjemi.uio.no and \\ Thomas Kjærgaard, qLEAP Center for Theoretical Chemistry, Department of Chemistry, Aarhus University, Langelandsgade 140, DK-8000 Aarhus C, Denmark. \\ Email: tkjaergaard@chem.au.dk}

An extension of the formulation of the atomic-orbital based response theory of Larsen et al., JCP 113, 8909 (2000) is presented. This new framework has been implemented in LSDalton and allows for the use of Kohn-Sham density-functional theory with approximate treatment of the Coulomb and Exchange contributions to the response equations via the popular resolution-of-the-identity approximation as well as the auxiliary-density matrix method (ADMM). We present benchmark calculations of ground-state energies as well as the linear and quadratic response properties: vertical excitation energies, polarizabilities and hyperpolarizabilities. The quality of these approximations in a range of basis sets is assessed against reference calculations in a large aug-pcseg-4 basis. Our results confirm that density fitting of the Coulomb contribution can be used without hesitation for all the studied properties. The ADMM treatment of exchange is shown to yield high accuracy for ground-state and excitation energies, whereas for polarizabilities and hyperpolarizabilities the performance gain comes at a cost of accuracy. Excitation energies of a tetrameric model consisting of units of the P700 special pigment of photosystem I have been studied to demonstrate the applicability of the code for a large system. 


\section{1 | INTRODUCTION}

In molecular electronic-structure theory, an essential step is the evaluation of two-electron integrals over one-electron basis functions. The explicit evaluation of these integrals comes at a high computational cost, and from the dawn of quantum chemistry, approximations have been introduced both to speed up molecular calculations and to reduce memory requirements [1]. Such approximate methods have been widely developed for the calculation of energies and gradients, but less attention has been given to developing these methods for the calculation of molecular properties.

The most widely used approach to approximate the Coulomb and exchange integrals is density fitting [2, 3, 4, 5, 6, 7, $8,9,10,11,12,13,14,15,16,17,18,19,20,21,22]$, also known as the resolution-of-the-identity (RI) approximation. In this approximation products of two one-electron basis functions are expanded in one-center auxiliary functions, and thus, the evaluation of four-center two-electron integrals is replaced by the evaluation of two- and three-center two-electron integrals and the solution of a set of linear equations. RI significantly improves performance with a limited impact on the accuracy and has therefore been applied to Hartree-Fock(HF)/Kohn-Sham(KS) theory, as well as correlated methods [22, 23, 24, 25, 26, 27]. An important alternative approach is the Cholesky-decomposition (CD) technique $[28,29,30,31,32]$ which to a large extent can be thought of as a special kind of density fitting where the auxiliary basis functions are obtained from the set of products between two one-electron basis functions through Cholesky-decomposition.

Combined with $J$-engine techniques $[33,34,35]$ RI gives tremendous speed-ups [8, 9] for Coulomb-like contributions. Although still applicable to exchange [10,11,12, 13, 16, 17, 20, 21], the RI methodology does not exhibit the same favorable performance gains as for the Coulomb integrals. Alternative schemes such as the auxiliary-density-matrix method (ADMM) [36, 37] and the chain-of-spheres algorithm (COSX) [38] have therefore been developed specifically for the exchange contribution. In ADMM, the exchange energy is split into two parts. One part consists of the exact $\mathrm{HF}$ exchange evaluated in a small auxiliary atomic basis set (from an auxiliary density matrix); the second part is a first-order correction term, evaluated as the difference between the generalized gradient approximation (GGA) exchange in the full and auxiliary basis sets. The auxiliary density matrix can be obtained by means of projection from the full density matrix fulfilling various constraints, as discussed by Guidon et al. [36] and Merlot et al. [37]. The COSX approximation builds on the use of semi-numerical integration techniques, first introduced by Friesner in the pseudo-spectral method [39, 40,41] and later refined in the COSX approach by Neese et al. [38]. In this approach the Coulomb potentials of products of two one-electron basis functions are evaluated analytically on a grid, followed by a numerical integration over the second electron. Reported speed-ups are of up to two orders of magnitude relative to calculations involving explicit exchange-matrix formation [38]. In this work we explore how these techniques may be exploited further in the calculation of molecular properties using response theory.

In response theory $[42,43,44,45,46,47,48,49,50]$ we determine the time development of an observable when the molecular system is subjected to an external electric or magnetic field. The frequency dependent perturbation causes the wave function to become frequency dependent. The response of the observable may be expanded in powers of the field strength: the linear response of the system is determined by the linear response function, the quadratic response by the quadratic response function, and so on [51]. The linear response function therefore represents the first-order correction to the expectation value of choice. The quadratic-response function represents the second order correction to the expectation value of choice and so on. For example, the frequency-dependent polarizability and hyper- 
polarizability may be evaluated from the linear and quadratic response functions, respectively. In the present work we investigate static (frequency independent) polarizabilities and hyperpolarizabilities. From the poles and residues of the response functions, additional molecular properties can be obtained, including vertical excitation energies to electronically excited states, strength parameters for (multi-photon) transitions to these states, and excited-state properties [42].

Recently, Ringholm et al. [52] presented an approach involving recursion for the open-ended calculation of response properties based on the density matrix-based quasi-energy formulation of the Kohn-Sham density functional response theory using perturbation- and time-dependent basis sets of Thorvaldsen et al. [45]. This approach was extended by Friese et al. to enable the calculation of single residues of response functions [53,54]. Very recently, this approach has furthermore been extended to include molecular environment effects by the polarizable embedding model [55].

The RI approximation has been extensively used in connection with CC2 molecular properties $[56,57,58,59,60$, $61,61,62,63,64,65]$, but has also been used in connection with the coupled perturbed Kohn-Sham equations[66]. It has also been used in TD-DFT in connection with excitation energies, excited state gradients and frequency-dependent optical rotation calculations $[67,68,69,70,71]$. Density fitting can also be applied for the efficient calculation of nuclear magnetic resonance shielding tensors [72, 73]. Ref. 67 and 70 concluded that the auxiliary basis sets developed for ground-state calculations are sufficient for most TD-DFT applications, although in some cases additional diffuse basis functions must be included. Ref. 70 reported that the total computational effort for excited-state optimizations is reduced by at least a factor of 4- 6 by the RI-J approximation, with corresponding RI-J errors of 0.01-0.02 eV. The RI-J errors in optimized bond lengths and angles amounted to less then $0.5 \mathrm{pm}$ and 1 degree, respectively. These deviations are usually much smaller than errors due to the incompleteness of the one-particle basis set.

Here we present a method for the computation of approximated response functions within the self-consistent-field (SCF) theories HF and KS density functional theory (DFT) and the extension to include ADMM, density fitting. The current formulation of approximate response theory presented in this work is general and works in principle for all approximations where the approximate Kohn-Sham matrix can be defined as the density matrix derivative of the approximate energy. It allows to easily accommodate future approximate methods that may differ from ADMM or RI in $\mathrm{HF} / \mathrm{KS}$ response theory.

The approximate response theory formulation introduced in this work is asymptotic linear scaling assuming that sparse matrix algebra is used. However, the focus of the present study is not on the scaling behavior of the approach with system size. Instead we use the new methodology to investigate the impact of the RI and ADMM approximations on the accuracy of linear and quadratic response properties. As examples we consider vertical excitation energies, static polarizabilities and static hyperpolarizabilities for a small benchmark consisting of 11 molecules, and vertical excitation energies for a tetramer P700 model system [74] consisting of 198 atoms. We commence in Section 2 by introducing the theoretical framework for the response calculations. Section 3 shows how this framework can easily accommodate the approximation techniques of the costly Coulomb and exchange integral contributions. Computational details are given in Section 4, and in Section 5 we present results for molecular response properties to assess the relative accuracy of these techniques in practical applications. Finally in Section 6 concluding remarks are drawn and directions for future work are discussed.

\section{2 | THEORY}

In order to provide a framework to easily introduce ADMM and other approximate methods in HF/KS response theory we first derive response theory in a formulation where the Fock/Kohn-Sham matrix takes a center stage. The derivation 
follows the response theory method of Larsen et al. [48] with a few modifications emphasizing the role of the Fock/KohnSham matrix, followed by an adaptation to ADMM and RI theories. The derivation assumes that all basis sets employed do not depend on the perturbation. Hence no London [75] (gauge including) atomic orbitals nor geometric perturbations are considered in the present work.

\section{1 | Time evolution of the Kohn-Sham system}

In Kohn-Sham (KS) density-functional theory (DFT) [76], the time evolution of the spin orbitals, in presence of a time dependent perturbation $V\left(\mathbf{r}_{1}, t\right)$, is governed by the time-dependent Schrödinger equation [77, 78, 79, 80],

$$
\left[f_{\mathrm{KS}}\left(\mathbf{r}_{1}, t\right)+V\left(\mathbf{r}_{1}, t\right)\right] \phi_{j}\left(\mathbf{r}_{1}, t\right)=i \frac{d \phi_{j}\left(\mathbf{r}_{1}, t\right)}{d t}
$$

where we have introduced the Kohn-Sham operator $f_{\mathrm{KS}}\left(\mathbf{r}_{1}, t\right)=h\left(\mathbf{r}_{1}\right)+j\left(\mathbf{r}_{1}, t\right)+v_{\mathrm{xc}}\left(\mathbf{r}_{1}, t\right)$, which we choose to define without the perturbation $V\left(r_{1}, t\right)$. The Kohn-Sham potential $f_{\mathrm{KS}}$ is defined as the functional derivative of the unperturbed energy functional

$$
f_{\mathrm{KS}}\left(\mathbf{r}_{1}, t\right)=\left.\frac{\delta E[\rho]}{\delta \rho\left(\mathbf{r}_{1}\right)}\right|_{\rho\left(\mathbf{r}_{1}\right)=\rho\left(\mathbf{r}_{1}, t\right)}
$$

which depends on the perturbation $V\left(r_{1}, t\right)$ through the density

$$
\rho(\mathbf{r}, t)=\sum_{\mu \nu} \chi_{\mu}^{*}(\mathbf{r}) \chi_{v}(\mathbf{r}) D_{\nu \mu}
$$

where $\mathbf{D}$ is the time dependent density matrix in the atomic-orbital (AO) basis and $\chi(\mathbf{r})$ denotes the AO basis functions. In the case of hybrid functionals $f_{\mathrm{KS}}$ may be supplemented by an orbital dependent term of the form, $w \cdot k\left(\mathbf{r}_{1}, \mathbf{r}_{2}, t\right)$, where $w$ is the weight of orbital dependent exchange and $k\left(r_{1}, r_{2}, t\right)$ is the derivative of the exchange energy with respect to the orbitals, as is used in Hartree-Fock theory.

Eq. (1) may be rewritten as a matrix equation using the expansion in $\mathrm{AOs} \chi_{\mu}$

$$
\phi_{i}\left(\mathbf{r}_{1}, t\right)=\sum_{\mu} C_{\mu i} \chi_{\mu}\left(\mathbf{r}_{1}\right)
$$

to obtain [48]

$$
\left(\mathbf{F}(\mathbf{D})+\mathbf{V}-i \mathbf{S} \frac{\partial}{\partial t}\right) \mathbf{C}=\mathrm{SC} \lambda
$$

with the constituents of the Kohn-Sham matrix $\mathbf{F}$, the time-dependent perturbation matrix $\mathbf{V}$ and the overlap matrix $\mathbf{S}$ are given in Eqs. (45-49) of the Appendix. Here $\lambda$ is a Hermitian Lagrange multiplier matrix. Eq. (5) reduces to the SCF equation in the perturbation free limit $(\mathbf{V}=0)$ and therefore time-independent limit $F(D) C=S C \lambda$.

Multiplying Eq. (5) with $\mathbf{C}^{T} \mathbf{S}$ from the right, subtracting the complex conjugate equation and introducing the density matrix in the $A O$ basis $D=C^{T}$ gives [48]

$$
(\mathbf{F}(\mathrm{D})+\mathrm{V}) \mathrm{DS}-\mathrm{SD}(\mathrm{F}(\mathrm{D})+\mathrm{V})=i \mathrm{SDS}
$$


Using the short hand notation $\dot{D}=\frac{\partial D}{\partial t}$ for the time derivative of the density matrix. Eq. (6) reduces to the standard stationary SCF condition $\mathbf{F D}_{0} \mathbf{S}-\mathbf{S D}_{0} \mathbf{F}=\mathbf{0}$ in the perturbation free limit, with $\mathbf{D}_{0}$ being the optimized $A O$ density matrix for the unperturbed system.

\section{2 | Response Equation}

The derivation of the AO-based response equations relies on an exponential parameterization of the density matrix and three expansions. The exponential parameterization of the density matrix, given by

$$
\mathbf{D}(\mathbf{X}(t))=\exp (-\mathbf{X}(t) \mathbf{S}) \mathbf{D} \exp (\mathbf{S X}(t))
$$

with $X(t)$ an anti-hermitian matrix, ensures that the symmetry, trace and idempotency conditions are imposed [81, 82], provided the reference density matrix $D$ also fulfills these conditions. The transformed density matrix $D(X)$, may be expanded using the the asymmetric Baker-Campbell-Hausdorff (BCH) expansion

$$
\mathbf{D}(\mathbf{X})=\mathbf{D}_{0}+[\mathbf{D}, \mathbf{X}]_{\mathbf{S}}+\frac{1}{2}[[\mathbf{D}, \mathbf{X}], \mathbf{X}]+\cdots,
$$

where the S-commutator is $[\mathbf{D}, \mathbf{X}]_{\mathbf{S}}=\mathbf{D S X}-\mathbf{X S D}$. The Kohn-Sham Fock matrix F may be Taylor expanded around the optimized $A O$ density matrix of the unperturbed state according to

$$
\begin{aligned}
\mathbf{F}(\mathbf{D}(\mathbf{X}))= & \mathbf{F}\left(\mathbf{D}_{0}\right)+\left.\frac{\partial \mathbf{F}}{\partial \mathbf{D}(\mathbf{X})}\right|_{\mathbf{D}(\mathbf{X})=\mathbf{D}_{0}}\left(\mathbf{D}(\mathbf{X})-\mathbf{D}_{0}\right) \\
& +\left.\frac{1}{2} \frac{\frac{\partial}{2}^{2} \mathbf{\partial}}{\partial \mathbf{D}(\mathbf{X})^{2}}\right|_{\mathbf{D}(\mathbf{X})=\mathbf{D}_{0}}\left(\mathbf{D}(\mathbf{X})-\mathbf{D}_{0}\right)^{2}+\cdots \\
= & \mathbf{F}\left(\mathbf{D}_{0}\right)+\mathbf{G}\left(\mathbf{D}(\mathbf{X})-\mathbf{D}_{0}\right)+\frac{1}{2} \mathbf{T}\left(\mathbf{D}(\mathbf{X})-\mathbf{D}_{0}, \mathbf{D}(\mathbf{X})-\mathbf{D}_{0}\right)+\cdots
\end{aligned}
$$

Here $\mathbf{G}(\mathbf{M})$ denotes the first derivative of the KS matrix contracted with a general matrix $M$

$$
\begin{aligned}
\mathbf{G}(\mathbf{M}) & =\left.\frac{\partial \mathbf{F}}{\partial \mathbf{D}(\mathbf{X})}\right|_{\mathbf{D}(\mathbf{X})=\mathbf{D}_{0}} \mathbf{M} \\
& =\mathbf{J}(\mathbf{M})+w \cdot \mathbf{K}(\mathbf{M})+\mathbf{K}^{\mathrm{XC}}(\mathbf{M}),
\end{aligned}
$$

with the full expressions for the Coulomb matrix $\mathrm{J}(\mathrm{M})$, exchange matrix $\mathrm{K}(\mathrm{M})$ and exchange correlation matrix $\mathrm{K}^{\mathrm{xc}}(\mathrm{M})$ given in Eq. (50) of the Appendix. For the second- (and higher-order) Kohn-Sham matrix derivatives only exchangecorrelation contributions remain

$$
T(\mathbf{N}, \mathbf{M})=\left.\frac{\partial^{2} \mathbf{F}(\mathbf{D}(\mathbf{X}))}{\partial \mathbf{D}(\mathbf{X})^{2}}\right|_{\mathbf{D}(\mathbf{X})=\mathbf{D}_{0}}(\mathbf{N}, \mathbf{M})=T^{\mathrm{xc}}(\mathbf{N}, \mathbf{M}),
$$

with $T^{\mathrm{xc}}(\mathbf{N}, \mathbf{M})$ given in Eq. (51) of the Appendix.

Finally, the set of parameters $\mathbf{X}(t)$ can be expanded in orders of the perturbation

$$
\mathbf{X}(t)=\mathbf{X}^{(1)}(t)+\mathbf{X}^{(2)}(t)+\cdots
$$


where the zero-order coefficients vanish since the reference state is optimized.

Inserting Eq. (8), Eq. (9) and Eq. (12) into Eq. (6), the parameters $\mathbf{X}^{(n)}(t)$ can now be determined by requiring the resulting equation to be valid to each order of the perturbation. The equation containing the first-order parameters $\mathbf{X}^{(1)}(t)$ is called the linear response equation, similarly the second-order parameters $\mathbf{X}^{(2)}(t)$ are obtained from the quadraticresponse equation and so forth.

\subsection{1 | Linear Response Equation}

The terms that contribute to the evaluation of the $\mathbf{X}^{(1)}(t)$ are

$$
\begin{aligned}
& \mathrm{G}\left(\left[\mathrm{D}, \mathbf{X}^{(1)}\right]_{\mathrm{S}}\right) \mathrm{DS}-\mathrm{SDG}\left(\left[\mathrm{D}, \mathbf{X}^{(1)}\right]_{\mathrm{S}}\right) \\
& +\mathrm{F}(\mathrm{D})\left[\mathrm{D}, \mathbf{X}^{(1)}\right]_{S} \mathrm{~S}-\mathrm{S}\left[\mathrm{D}, \mathbf{X}^{(1)}\right]_{S} \mathrm{~F}(\mathrm{D}) \\
& +\mathrm{VDS}-\mathrm{SDV}=i \mathrm{~S}[\mathrm{D}, \dot{\mathrm{X}}]_{\mathrm{S}} \mathrm{S}
\end{aligned}
$$

This first-order equation can be solved using the Fourier expansion [48] of $X^{(1)}(t)$,

$$
X^{(1)}(t)=\int_{-\infty}^{\infty} \exp (-i \omega t) X^{(1)}(\omega) d \omega
$$

to obtain:

$$
\begin{aligned}
& \mathbf{G}\left(\left[\mathbf{D}, \mathbf{X}^{(1)}(\omega)\right]_{S}\right) \mathbf{D S}-\mathbf{S D G}\left(\left[\mathbf{D}, \mathbf{X}^{(1)}(\omega)\right]_{S}\right) \\
& +\mathbf{F}(\mathbf{D})\left[\mathbf{D}, \mathbf{X}^{(1)}(\omega)\right]_{S} \mathbf{S}-\mathbf{S}\left[\mathbf{D}, \mathbf{X}^{(1)}(\omega)\right]_{S} \mathbf{F}(\mathbf{D}) \\
& +\mathrm{VDS}-\mathbf{S D V}=-\omega \mathbf{S}[\mathbf{D}, \mathbf{X}(\omega)]_{S} S
\end{aligned}
$$

It can also be written in the shorthand notation $[83,49]$

$$
\left(\mathbf{E}^{[2]}-\omega \mathbf{S}^{[2]}\right) \mathbf{X}(\omega)=\mathbf{V D S}-\mathbf{S D V}
$$

with the generalized Hessian $\mathbf{E}^{[2]}$ defined through the transformation

$$
\begin{aligned}
& \mathbf{E}^{[2]} \mathbf{X}(\omega)=-\mathbf{G}\left(\left[\mathbf{D}, \mathbf{X}^{(1)}(\omega)\right]_{\mathbf{S}}\right) \mathbf{D S}+\mathbf{S D G}\left(\left[\mathbf{D}, \mathbf{X}^{(1)}(\omega)\right]_{S}\right) \\
& -\mathbf{F}\left[\mathbf{D}, \mathbf{X}^{(1)}(\omega)\right]_{\mathbf{S}} \mathbf{S}+\mathbf{S}\left[\mathbf{D}, \mathbf{X}^{(1)}(\omega)\right]_{\mathbf{S}} \mathbf{F}
\end{aligned}
$$

and the generalized metric matrix $\mathbf{S}^{[2]}$ through

$$
\mathbf{S}^{[2]} \mathbf{X}(\omega)=\mathbf{S}[\mathbf{D}, \mathbf{X}(\omega)]_{\mathbf{S}} \mathbf{S}
$$

Although the frequency is not necessary for the static properties considered in this paper, we include it in the following theory for generality. The excitation energies are identified as the poles of the linear response equation and are therefore solutions to the generalized eigenvalue problem given by

$$
\mathbf{E}^{[2]} \mathbf{X}_{f}=\mathbf{S}^{[2]} \mathbf{X}_{f} \omega_{f}
$$


Similarly, for the evaluation of $\mathbf{X}^{(2)}(t)$, the quadratic response equation becomes,

$$
\begin{aligned}
& \left(\mathbf{E}^{[2]}-\left(\omega_{1}+\omega_{2}\right) \mathbf{S}^{[2]}\right) \mathbf{X}^{(2)}\left(\omega_{1}, \omega_{2}\right)= \\
& \mathbf{V}\left[\mathbf{D}, \mathbf{X}^{(2)}\left(\omega_{1}, \omega_{2}\right)\right]_{\mathbf{S}} \mathbf{S}-\mathbf{S}\left[\mathbf{D}, \mathbf{X}^{(2)}\left(\omega_{1}, \omega_{2}\right)\right]_{\mathbf{S}} \mathbf{V} \\
& -P_{12}\left(\mathbf{E}^{[3]}-\left(\omega_{1}+\omega_{2}\right) \mathbf{S}^{[3]}\right) \mathbf{X}^{(1)}\left(\omega_{1}\right) \mathbf{X}^{(1)}\left(\omega_{2}\right) .
\end{aligned}
$$

The terms contributing to the quadratic response equation are given in Eq. (52) of the Appendix.

\section{3 | Response Functions}

Response functions describe the corrections to the expectation value of a Hermitian operator $\hat{A}$, representing an observable, due to the perturbation

$$
\begin{aligned}
\langle\tilde{0}|\hat{A}| \tilde{0}\rangle & =\langle 0|\hat{A}| 0\rangle \\
& +\int_{-\infty}^{\infty}\langle\langle A ; V(\omega)\rangle\rangle_{\omega} \exp [(-\mathrm{i} \omega+\epsilon) t] \mathrm{d} \omega \\
& +\frac{1}{2} \iint_{-\infty}^{\infty}\left\langle\left\langle A ; V\left(\omega_{1}\right), V\left(\omega_{2}\right)\right\rangle\right\rangle_{\omega_{1}, \omega_{2}} \\
& \exp \left[\left(-\mathrm{i}\left(\omega_{1}+\omega_{2}\right)+2 \epsilon\right) t\right] \mathrm{d} \omega_{1} \mathrm{~d} \omega_{2}+\ldots
\end{aligned}
$$

Using Eq. (8) to parameterize the expectation value

$$
\begin{aligned}
& \operatorname{Tr}(\mathbf{A D}(\mathbf{X}))=\operatorname{Tr}\left(\mathbf{A} \mathbf{D}_{0}\right)+\operatorname{Tr}\left(\mathbf{A}[\mathbf{D}, \mathbf{X}]_{\mathbf{S}}\right) \\
& +\frac{1}{2} \operatorname{Tr}(\mathbf{A}[[\mathbf{D}, \mathbf{X}], \mathbf{X}])+\cdots
\end{aligned}
$$

the Fourier expansion of X, Eq. (14), and collecting orders of the perturbation, one obtains the linear response function

$$
\langle\langle A ; V(\omega)\rangle\rangle_{\omega}=\operatorname{Tr}\left(\mathbf{A}\left[\mathbf{D}, \mathbf{X}^{(1)}\right] \mathbf{S}\right),
$$

and the quadratic response function

$$
\begin{aligned}
\left\langle\left\langle A ; V\left(\omega_{1}\right),\right.\right. & \left.\left.V\left(\omega_{2}\right)\right\rangle\right\rangle_{\omega_{1}, \omega_{2}}=\operatorname{Tr}\left(\mathbf{A}\left[\mathbf{D}, \mathbf{X}^{(2)}\right] \mathbf{s}\right) \\
+ & \frac{1}{2} \operatorname{Tr}\left(\mathbf{A}\left[\left[\mathbf{D}, \mathbf{X}^{(1)}\left(\omega_{1}\right)\right], \mathbf{X}^{(1)}\left(\omega_{2}\right)\right]\right) \\
& +\frac{1}{2} \operatorname{Tr}\left(\mathbf{A}\left[\left[\mathbf{D}, \mathbf{X}^{(1)}\left(\omega_{2}\right)\right], \mathbf{X}^{(1)}\left(\omega_{1}\right)\right]\right) .
\end{aligned}
$$

\section{3 | RESPONSE THEORY WITH APPROXIMATE INTEGRALS}

When using approximations like density fitting or ADMM the energy and Kohn-Sham matrix change. This means that the Taylor expansion of the Kohn-Sham matrix must also change and thus a modified $\mathbf{G}$ and/or $\mathbf{T}$ is required in place of Eq. (10) and (11). However, everything else remains the same. The response functions can still be evaluated from Eq. (23) and (24) with response parameters obtained by solving the response equations in Eq. (16) and Eq. (20). Excitation 
energies and excitation eigenvectors can still be determined from Eq. (19).

In this section we derive expressions for $\mathbf{G}$ and $\mathbf{T}$ for density fitting and the auxiliary density matrix method (ADMM). This framework is general and can be applied to all approximations where the approximate Kohn-Sham matrix can be defined as the density matrix derivative of the approximate energy. This provides a clear road map for the implementation of response theory using both present and future approximations that accelerate the evaluation of contributions arising from the Coulomb and exchange integrals.

\section{1 | Kohn Sham matrix expansion using density fitting}

In density fitting products of two one-electron functions are expanded in auxiliary atom-centered functions $I(\mathbf{r})$, according to

$$
|\mu v\rangle \approx|\widetilde{\mu v}\rangle=\sum_{I} c_{I}^{\mu v}|I\rangle
$$

with indices $\mu, \nu, \rho, \sigma$ denoting the primary AO basis functions, in order to avoid the expensive evaluation of the fourcenter two-electron integrals $(\mu v \mid \rho \sigma)$. Instead only two- and three-center integrals need to be evaluated, at the expense of solving a set of linear equations for the coefficients $c_{I}^{\mu \nu}$. The integrals, given in Mulliken notation,

$$
(\mu v \mid \rho \sigma)=\iint \chi_{\mu}\left(\mathbf{r}_{1}\right) \chi_{\nu}\left(\mathbf{r}_{1}\right) r_{12}^{-1} \chi_{\rho}\left(\mathbf{r}_{2}\right) \chi_{\sigma}\left(\mathbf{r}_{2}\right) d \mathbf{r}_{1} d \mathbf{r}_{2},
$$

can be approximated in different ways, for example according to the three term expansion [15]

$$
(\mu v \mid \rho \sigma) \approx \widetilde{(\mu v \mid \rho \sigma})=(\widetilde{\mu v} \mid \rho \sigma)+(\mu v \mid \widetilde{\rho \sigma})-(\widetilde{\mu v} \mid \widetilde{\rho \sigma}),
$$

where the two first terms involve three-center integrals and the last term involves two-center integrals. The different flavors of density fitting arise from the choice of 1) the set of auxiliary functions $\{I\}$ included in the expansion of Eq. (25), 2) how the fitting coefficients $c_{I}^{\mu \nu}$ are obtained and 3) the ansatz for the integral approximation Eq. (27). The set of functions $\{I\}$ can range from including only auxiliary functions centered on the two parent atoms [4] of $\mu$ and $v$ to the full set of auxiliary functions on all atoms in the molecule $[5,6]$. The fitting coefficients are obtained by minimizing the error $\Delta_{\mu \nu}^{w}$ of the residual density $\left.\left|\delta_{\mu \nu}\right\rangle=|\mu \nu\rangle-\widetilde{\mu v}\right\rangle$ in metric $w$

$$
\Delta_{\mu \nu}^{w}=\left\langle\delta_{\mu \nu}|w| \delta_{\mu \nu}\right\rangle
$$

where $w$ can range $[12,14]$ from the Coulomb operator $r_{12}^{-1}$ to the Dirac delta function (overlap metric fitting), and where the minimization can be subjected to charge, dipole or higher-order constraints. The three term ansatz of Eq. (27) is denoted robust [15] in the sense that it ensures that the errors in the fitted integrals are bilinear with respect to the errors in the two-center fits,

$$
(\mu v \mid \rho \sigma)-\widetilde{(\mu v \mid \rho \sigma)}=\left(\delta_{\mu v} \mid \delta_{\rho \sigma}\right)
$$


whereas including for instance only the first term has a linear error. We note that when doing the standard (unconstrained) Coulomb-metric fitting over the full set of auxiliary functions, giving the fitting equation set

$$
\sum_{\beta}(I \mid J) c_{J}^{\mu v}=(I \mid \mu v)
$$

and similarly for $(\rho \sigma)$, the error is bilinear using only the first, second or third (with a positive sign) term of Eq. (27), as it follows from Eq. (30) that $(\widetilde{\mu v} \mid \rho \sigma)=(\mu v \mid \widetilde{\rho \sigma})=(\widetilde{\mu v} \mid \widetilde{\rho \sigma})$.

When considering external perturbations, one needs to include Lagrangian terms in the integral approximation to ensure that the equations for the fitting coefficients are satisfied with respect to the perturbation. In this paper we only consider perturbations that do not affect the fitted integrals, and the Lagrangian formalism therefore does not need to be considered here. Hence, the application of response theory is straightforwardly achieved by a simple replacement of the exact integrals $(\mu v \mid \rho \sigma)$ with the fitted integrals $(\widetilde{\mu v \mid \rho \sigma})$. For the standard density-fitting approximation we simply replace the exact expressions of $\mathrm{J}(\mathrm{M})$ and $\mathrm{K}(\mathrm{M})$ in Eq. (10), with the approximate expressions $\tilde{J}(\mathrm{M})$ and $\tilde{\mathrm{K}}(\mathrm{M})$, given by

$$
\begin{aligned}
\tilde{\mathbf{J}}(\mathrm{M}) & \left.=\sum_{\rho \sigma} \widetilde{(\mu v \mid \rho \sigma}\right) M_{\rho \sigma}=\sum_{I}(\mu v \mid I) c_{I}, \\
\tilde{\mathbf{K}}(\mathbf{M}) & =\sum_{\rho \sigma} \widetilde{(\mu \sigma \mid \rho v)} M_{\rho \sigma}=\sum_{I} \sum_{\rho \sigma}(\mu \sigma \mid I) c_{I}^{\rho v} M_{\rho \sigma} \\
c_{I} & \equiv \sum_{\rho \sigma} c_{I}^{\rho \sigma} M_{\rho \sigma}=(I \mid J)^{-1} \sum_{\rho \sigma}(J \mid \rho \sigma) M_{\rho \sigma}
\end{aligned}
$$

to obtain

$$
\tilde{\mathbf{G}}(\mathbf{M})=\tilde{\mathbf{J}}(\mathbf{M})+w \cdot \tilde{\mathbf{K}}(\mathbf{M})+\mathbf{K}^{\mathrm{xc}}(\mathbf{M})
$$

where density fitting is used for both Coulomb and exchange. Note, that whereas three-index coefficients $c_{I}^{\rho \nu}$, or variants thereof $[10,11,13,16,17,20,21]$, are needed for the exchange contribution, only the one-index coefficients $c_{I}$ are needed for the Coulomb contribution. Additionally, for the Coulomb contribution density fitting can be combined with $J$-engine techniques [8, 9], and as a result greatly outperform the exchange version, thus motivating the search for alternative approximative approaches for the exchange contribution. In this paper we will consider density fitting for the Coulomb contribution, and investigate the effects of one exchange alternative, the ADMM approach, which is outlined in the next section.

We note finally that approximate Fock/KS matrix construction that requires a decomposition of the density matrix, such as using (local) molecular orbitals[30, 84], cannot straightforwardly be applied in the response framework presented in this paper, as the density matrix dependence changes with the external perturbation.

\subsection{Kohn Sham matrix expansion using ADMM}

The expression for the ADMM energy is based on the following trivial rearrangement of the total exchange energy $E^{K}$ :

$$
E^{K}(\mathbf{D})=E^{k}(\mathbf{d})+E^{K}(\mathbf{D})-E^{k}(\mathbf{d}),
$$


where upper-case letters denote quantities evaluated in the primary basis and lower-case letters quantities in the auxiliary basis. $\mathbf{D}$ is the density matrix in the primary atomic-orbital (AO) basis and $\mathbf{d}$ is a density matrix obtained by projection of $D$ to some (smaller) auxiliary electron density. The ADMM exchange energy $\left(E_{\mathrm{admm}}^{K}\right)$ is obtained by replacing the exact-exchange terms $E^{K}(\mathbf{D})-E^{k}(\mathrm{~d})$ in Eq. (33) with exchange functionals $E_{\mathrm{admm}}^{\mathrm{x}}[\rho]-E_{\mathrm{admm}}^{\mathrm{x}}\left[\rho_{\text {admm }}\right]$ to give

$$
\begin{aligned}
E_{\mathrm{admm}}^{K}(\mathbf{D}) & =E^{k}(\mathbf{d})+E_{\mathrm{admm}}^{\mathrm{x}}[\rho]-E_{\mathrm{admm}}^{\mathrm{x}}\left[\rho_{\mathrm{admm}}\right] \\
& =\frac{1}{2} \sum_{\alpha \beta \gamma \delta} d_{\alpha \beta}(\alpha \beta \mid \gamma \delta) d_{\gamma \delta}+\int_{\mathbb{R}^{3}} \epsilon_{\mathrm{x}}[\rho] \mathrm{dr} \\
& -\int_{\mathbb{R}^{3}} \epsilon_{\mathrm{x}}\left[\rho_{\mathrm{admm}}\right] \mathrm{dr},
\end{aligned}
$$

where the Greek letters $\alpha, \beta, \gamma, \delta$ denote auxiliary AOs, and $\rho$ the electron density

$$
\rho(\mathbf{r})=\sum_{\rho \sigma} \chi_{\rho}(\mathbf{r}) \chi_{\sigma}(\mathbf{r}) D_{\rho \sigma}
$$

and $\rho_{\text {admm }}$ the auxiliary density

$$
\rho_{\mathrm{admm}}(\mathbf{r})=\sum_{\alpha \beta} \chi_{\alpha}(\mathbf{r}) \chi_{\beta}(\mathbf{r}) d_{\alpha \beta}
$$

Note, that the exchange functional used in the ADMM approximation (denoted with $\mathrm{x}$ and admm) may be different from the exchange part of the exchange-correlation functional employed (denoted with $\mathrm{xc}$ ).

Here we focus on the ADMM2 approximation [36] where the auxiliary density matrix $\mathbf{d}$ is obtained through projection, and can be written in terms of the regular $A O$ density matrix $D$ as

$$
\mathbf{d}=\mathbf{W D} \mathbf{W}^{T}, \mathbf{W}=\mathbf{s}^{-1} \mathbf{Q}
$$

where $\mathbf{s}$ is the $\mathrm{AO}$ overlap matrix in the auxiliary basis with elements $s_{\alpha \beta}=\int \chi_{\alpha}\left(\mathbf{r}_{1}\right) \chi_{\beta}\left(\mathbf{r}_{1}\right) \mathrm{d} \mathbf{r}_{1}$; and $\mathbf{Q}$ is the mixed auxiliary-primary AO overlap matrix with elements $Q_{\alpha \mu}=\int \chi_{\alpha}\left(\mathbf{r}_{1}\right) \chi_{\mu}\left(\mathbf{r}_{1}\right) \mathrm{dr} \mathbf{r}_{1}$. It follows that the ADMM2 exchange matrix is given by

$$
\begin{aligned}
K_{\mu \nu}^{\mathrm{admm}}(\mathbf{D}) & =\frac{\partial E_{\mathrm{admm}}^{K}}{\partial D_{\mu \nu}} \\
& =\mathbf{F}_{\mu \nu}^{\mathrm{x}, \mathrm{admm}}(\mathbf{D})+\mathbf{W}^{T}\left(\mathbf{k}(\mathbf{d})-\mathbf{f}^{\mathrm{x}, \mathrm{admm}}(\mathbf{d})\right) \mathbf{W},
\end{aligned}
$$

and its resulting derivatives can be expressed as

$$
\begin{aligned}
\frac{\partial \mathbf{K}^{\mathrm{admm}}(\mathrm{D})}{\partial \mathbf{D}} \mathbf{M} & =\mathbf{K}^{\mathrm{x}, \mathrm{admm}}(\mathbf{M}) \\
& +\mathbf{W}^{T}\left(\mathbf{k}(\mathbf{m})-\mathbf{K}^{\mathrm{x}, \mathrm{admm}}(\mathbf{m})\right) \mathbf{W} \\
\frac{\partial^{2} \mathbf{K}^{\mathrm{admm}}(\mathbf{D})}{\partial \mathbf{D} \partial \mathbf{D}} \mathbf{M N} & =\mathbf{T}^{\mathrm{x}, \mathrm{admm}}(\mathbf{M}, \mathbf{N}) \\
& -\mathbf{W}^{T}\left(\mathbf{T}^{\mathrm{x}, \mathrm{admm}}(\mathbf{m}, \mathbf{n})\right) \mathbf{W}
\end{aligned}
$$


with

$$
\mathbf{m}=\mathbf{W M W}^{T}, \quad \mathbf{n}=\mathbf{W N W}^{T} .
$$

Due to the two exchange-functional terms in the ADMM approximation, the exchange contribution will, like the exchange-correlation contribution, have non-vanishing terms for all derivative orders. Here however we limit ourself to quadratic response and thus only up to second order. Using the ADMM2 approximation leads to the following modified expressions for the derivative contributions

$$
\begin{aligned}
\mathbf{G}^{\mathrm{admm}}(\mathbf{M}) & =\mathbf{J}(\mathbf{M})+w \mathbf{K}^{\mathrm{x}, \mathrm{admm}}(\mathbf{M}) \\
& +w \mathbf{W}^{T}\left(\mathbf{k}(\mathbf{m})-\mathbf{K}^{\mathrm{x}, \mathrm{admm}}(\mathbf{m})\right) \mathbf{W} \\
& +\mathbf{K}^{\mathrm{xC}}(\mathbf{M}) \\
\mathbf{T}^{\mathrm{admm}}(\mathbf{M}, \mathbf{N}) & =w \mathbf{T}^{\mathrm{x}, \operatorname{admm}}(\mathbf{M}, \mathbf{N}) \\
& -w \mathbf{W}^{T}\left(\mathbf{T}^{\mathrm{X}, \mathrm{admm}}(\mathbf{m}, \mathbf{n})\right) \mathbf{W}+\mathbf{T}^{\mathrm{Xc}}(\mathbf{M}, \mathbf{N})
\end{aligned}
$$

replacing Eq.(10) and (11). Combining density fitting for Coulomb and ADMM for exchange is straightforwardly achieved by substituting $\mathbf{J}(\mathbf{M})$ with $\tilde{\mathbf{J}}(\mathbf{M})$ for the $\mathbf{G}$ term of Eq. (41), whereas the $\mathbf{T}$ term remains unchanged. Note that for computational efficiency the ADMM exchange-functional contributions in the regular AO basis can be calculated together with the exchange-correlation contribution simply by augmenting the exchange-correlation functional with the additional ADMM exchange functional terms.

We finally note that developing AO response theory for the flavors of ADMM presented in Ref. [37], ADMMQ, ADMMS and ADMMP, follows the same general principles. However, the expressions become more involved; secondorder derivatives require the first-order derivative of the scaling factor $\xi$ and third-order derivatives require the first-order derivative of both the scaling factor $\xi$ and the Lagrangian multiplier $\Lambda$, in accordance with the $2 n+1$ and $2 n+2$ rules for variational and Lagrangian parameters, respectively [? ]. For the ADMM1 and ADMM3 flavors of Ref. [36] an AO-based response formulation is not straightforwardly obtained. ADMM1 is MO-based and there is no clear way to differentiate with respect to the $A O$ density-matrix elements. For $A D M M 3$, the same holds when a density-matrix purification method is used.

\section{4 | COMPUTATIONAL DETAILS}

The accuracy of the density fitting and ADMM approximations has been tested on a set 11 molecules for electronic ground state energies, vertical excitation energies, static polarizabilities and first hyperpolarizabilities. For the vertical excitation energies, the five lowest excitations were considered. The methods under investigation have been run with a local development version of LSDalton [85, 86] on single nodes of a $2.6 \mathrm{GHz}$ Intel E5-2670 cluster, employing OpenMP to utilize the 16 cores on each node.

The test set has been chosen from Ref. [87]. The molecules investigated are acetamide $\left(\mathrm{C}_{2} \mathrm{H}_{5} \mathrm{ON}\right)$, acetone $\left(\mathrm{C}_{3} \mathrm{H}_{6} \mathrm{O}\right)$, butadiene $\left(\mathrm{C}_{4} \mathrm{H}_{6}\right)$, cyclopropene $\left(\mathrm{C}_{3} \mathrm{H}_{4}\right)$, ethene $\left(\mathrm{C}_{2} \mathrm{H}_{4}\right)$, formaldehyde $\left(\mathrm{CH}_{2} \mathrm{O}\right)$, formamide $\left(\mathrm{CH}_{3} \mathrm{ON}\right)$, furan $\left(\mathrm{C}_{4} \mathrm{H}_{4} \mathrm{O}\right)$, imidazole $\left(\mathrm{C}_{3} \mathrm{H}_{4} \mathrm{~N}_{2}\right)$, propanamide $\left(\mathrm{C}_{3} \mathrm{H}_{7} \mathrm{ON}\right)$ and pyrrole $\left(\mathrm{C}_{4} \mathrm{H}_{5} \mathrm{~N}\right)$. This set of molecules will in the following be denoted as M11.

For each property, the basis set performance of three different types of calculations labeled as full, $d f-J$ and $a d m m$ have been investigated. Here "full" are regular calculations employing standard $J$-engine techniques $[33,34,35]$ for the 
Coulomb contribution and LinK [88] for the exchange contribution, i.e. without any approximation (except standard acceleration techniques and integral pre-screening), "df- $J$ " refers to the combination of density fitting for Coulomb and LinK for exchange, and "admm" to the combination of density fitting for Coulomb and ADMM (ADMM2) for exchange.

For the basis set performance, we have chosen the Jensen DFT optimized pcseg- $n$ and aug-pcseg- $n$ basis sets [89] as orbital basis, and have investigated the accuracy for cardinal numbers $n=1,2,3$ against reference aug-pcseg-4 calculations. The prefix "aug" indicates the use of augmented functions. For density fitting the Karlsruhe def2-QZVPP auxiliary basis set of Weigend has been employed[90], for pcseg- $n$ ADMM calculations the auxiliary admm- $n$ basis sets [91] have been employed, and for the aug-pcseg- $n$ ADMM calculations the aug-admm- $n$ basis sets have been employed. The admm- $n$ basis sets have been specifically optimized for the ADMM approximation to be used along with the pcseg- $n$ family of basis sets. The ADMM basis set is developed for elements up to period 3 excluding noble gas elements. The aug-admm- $n$ basis sets have been adapted by augmenting the admm- $n$ basis sets using the augmented functions from the aug-pcseg- $n$ basis sets with cardinal number $n-1$. The optimized ADMM basis sets are available from a git repository upon request.[92]

In this paper we employ the CAM-B3LYP functional with the specifications $\alpha=0.21, \beta=0.79$ and $\mu=0.45$, rather than the standard CAM-B3LYP functional parameters $\alpha=0.19, \beta=0.46$ and $\mu=0.33$. The main motivation of this choice is to include full long-range exchange treatment, with the aim to 1) push the errors of the ADMM exchange approximation to its limit, and 2) treat larger systems. For ADMM we have constructed a GGA correction functional, given by Eq. (59) in section 9.4 of the appendix. This GGA correction functional is employed for all calculations involving CAM-B3LYP, with the same functional parameters specifications of $\alpha, \beta$ and $\mu$ as the CAM-B3LYP functional used in the calculation. For the benchmarks studied here, the molecular size is limited by the time-demanding aug-pcseg- 4 reference LinK calculations (the reference calculation on propanamide took roughly 3 weeks to complete). The chosen CAM-B3LYP parameterization is expected to perform well for the properties considered, in particular for polarizabilities and first hyperpolarizabilities, but also for the investigated excitation energies.

As an example application for a larger system, we have calculated the five lowest singlet vertical excitation energies of a tetrameric P700 model, taken from Ref.[74]. The model system is depicted in Figure 11. For these calculations we have employed a hybrid MPI/OpenMP parallelisation scheme, employing 36 nodes each with two 16 -core $2.1 \mathrm{GHz}$ Intel Broadwell chips; for a total of 1024 cores.

\section{5 | RESULTS AND DISCUSSION}

Rather than investigating the errors of each method in a given basis set, we have chosen to make a comparison against the basis-set limit. This choice has been taken in order to assess how the methods perform in typical practical calculations. In the following a graphical summary is presented, visualizing normal distributions

$$
f(x)=N \exp \left(-\frac{(x-\mu)^{2}}{2 \sigma^{2}}\right)
$$

using the mean error $\mu$ and the standard deviation $\sigma$ for each employed approximation, property and basis set. Using the normalization $N$ makes a visual comparison of the differences among methods and basis sets straight forward. For electronic ground-state energies we have used the normalization $N=\log \left(\frac{1}{\sqrt{2 \sigma^{2} \pi}}\right)$, and for excitation energies, isotropic polarizabilities, anisotropic polarizabilities and dipole hyperpolarizability $N=\frac{1}{\sqrt[4]{2 \sigma^{2} \pi}}$. For the figures we have chosen to represent only the "df- $J$ " and "admm" results, since the "full" and "df- $J$ " results are virtually identical, see supplementary information for detailed results including all three types of calculations. In the supplementary information we have also 


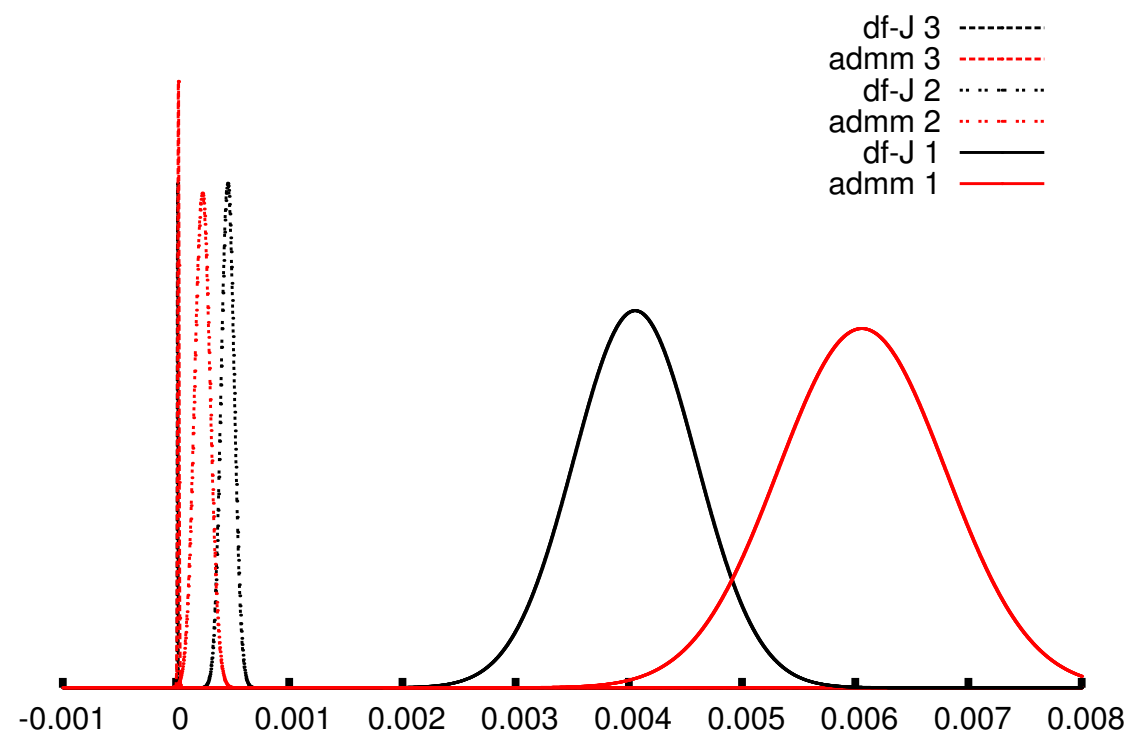

FIGURE 1 The normal distribution of errors in the molecular ground state energy (in atomic units) with CAM-B3LYP $(\alpha=0.21, \beta=0.79, \mu=0.45) / p c s e g-n(n=1,2,3)$ for a set of molecules M11 (see text). Here aug-pcseg- 4 results are taken as the reference.

included maximum absolute errors. These errors do not change the overall picture of the results. As such, we will not discuss maximum absolute errors here, but instead refer the interested reader to the results given in the supplementary information. In the figures, the labels "df- $J n$ " and "admm $n$ " denote a df- $J$ and admm type calculation, respectively, employing the pcseg- $n$ basis sets, and similarly "df- $J$ aug- $n$ " and "admm aug- $n$ " where the aug-pcseg- $n$ basis sets are employed. In the following we use capital letter ADMM for the ADMM approximation and lower case letters admm for the calculations using both $\mathrm{df}-J$ and ADMM.

\section{1 | Electronic ground-state energy}

In Figure 1 the normal distributions of the errors in the electronic ground-state energy per electron for pcseg- $n$, $n=1,2,3$, in reference to aug-pcseg-4, are plotted for $\mathrm{df}-J$ and admm calculations. We emphasize again that the full and the df- $J$ results are very similar and we have therefore chosen to present only one of the two in the plots. The results for all three methods are given in the supplementary information Table S1. Some representative example numbers are presented and discussed in the text below.

While the variance (width) is rather large for both methods when a pcseg-1 basis set is employed a clear improvement is seen upon increasing the basis set to pcseg-2 and pcseg-3; the errors are reduced by about one order of magnitude with each cardinal number $n$, for all methods. On average all methods investigated overestimate the ground state energy compared to the aug-pcseg-4 reference, which is to be expected from any variational approach. For example, the mean errors (standard deviations) in a pcseg-2 basis are 458(58), 456(58) and 232(69) $\mu \mathrm{E}_{H}$ for full, df- $J$ and admm type calculations, respectively, while for the pcseg-3 basis these values decrease to $17(2), 14(2)$ and $21(6)$ 


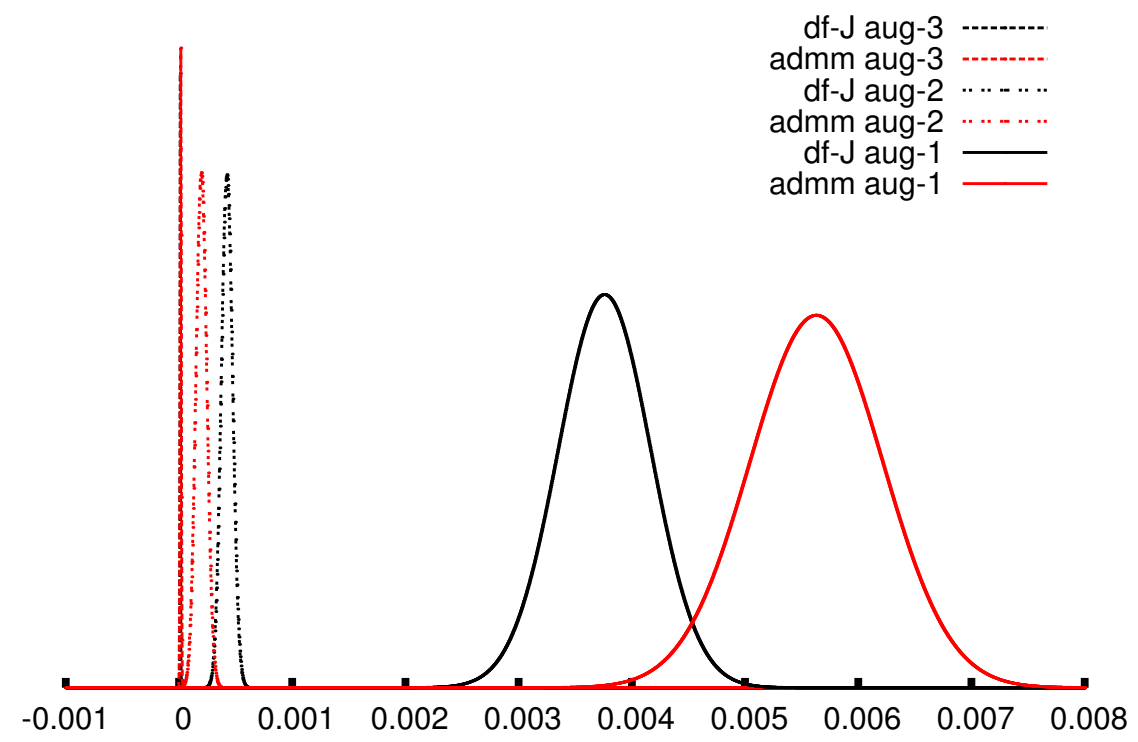

FIGURE 2 The normal distribution of errors in the molecular ground state energy (in atomic units) with CAM-B3LYP ( $\alpha=0.21, \beta=0.79, \mu=0.45)$ /aug-pcseg- $(n=1,2,3)$ for a set of molecules M11 (see text). Here aug-pcseg-4 results are taken as the reference.

$\mu \mathrm{E}_{H}$; all of which are well below $1 \mathrm{kcal} / \mathrm{mol}$ (or $1594 \mu \mathrm{E}_{H}$ ). Although the mean error for admm at the pcseg-2 level is smaller than for the df- $J$ calculation by almost a factor two, it is about $50 \%$ larger in the other two cases, and the standard deviation for admm is larger in each case.

In Figure 2 the corresponding errors in the electronic ground-state energies are depicted for aug-pcseg- $n, n=1,2,3$. As expected, augmentation leads to some improvement (reducing the errors by $10-30 \%$ ), but does not change the observed trends.

\section{2 | Vertical excitation energies}

Figures 3 and 4 show the normal distributions of the absolute errors in the five lowest vertical excitation energies of the M11 benchmark set for $\mathrm{df}-J$ and admm type calculations using pcseg- $n$ and aug-pcseg- $n$ basis sets, with $n=1,2,3$, respectively. The 55 excitation energies considered here are in the range $3.93 \mathrm{eV}$ to $8.96 \mathrm{eV}$ at the reference aug-pcseg- 4 full level of theory. The calculations have been run without any point group symmetry, and no attempt has been made to identify the different states. Thus, the order of the excitation energies may vary, in particular for nearly degenerate states, at different basis set and theory levels.

Similar to the trend for the electronic ground-state energies, the excitation energies are overestimated as compared to the aug-pcseg-4 reference. All methods show a systematic improvement with increasing cardinal number $n$, and at each level $n$, the full and df- $J$ type calculations perform better than admm. Upon augmentation the errors get reduced by one to two orders of magnitude, and already at the aug-pcseg-1 basis set level the errors are smaller that the corresponding pcseg-3 results; with errors 49(91) meV, 49(91) meV and 34(124) meV, for full, df- $J$ and admm type 


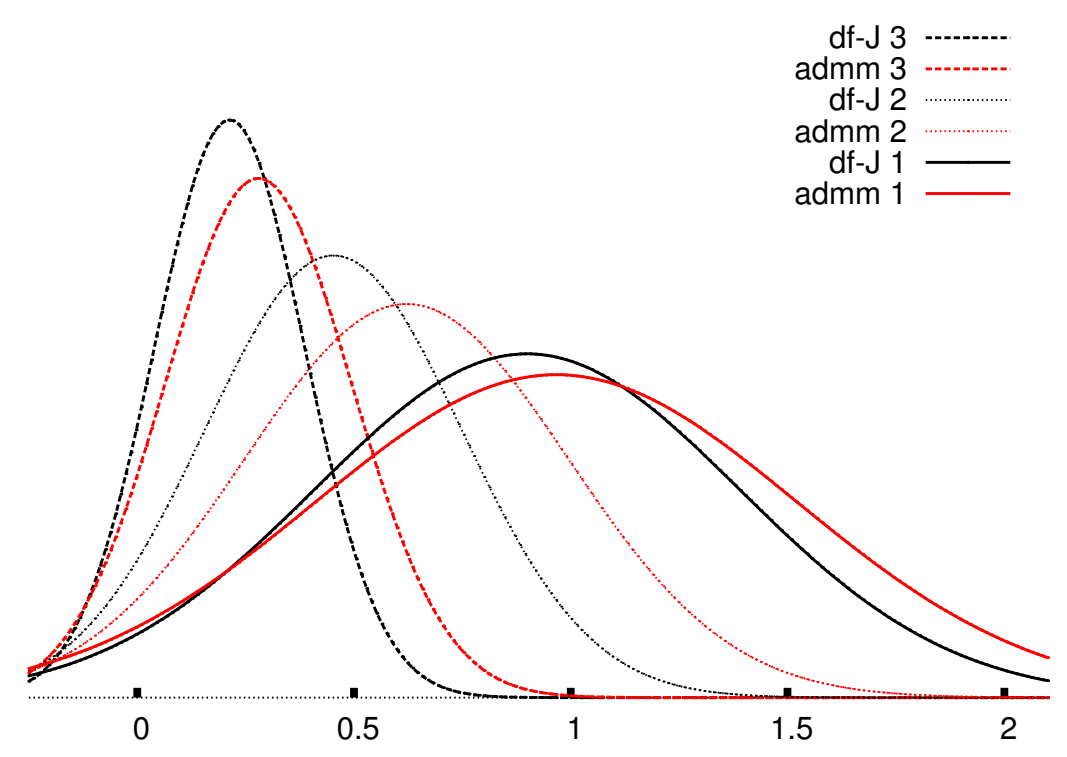

FIGURE 3 The normal distribution of errors in the first five excitation energies (in electron volt) with CAM-B3LYP $(\alpha=0.21, \beta=0.79, \mu=0.45) / p c s e g-n(n=1,2,3)$ for a set of molecules M11 (see text). Here aug-pcseg-4 results are taken as the reference. 


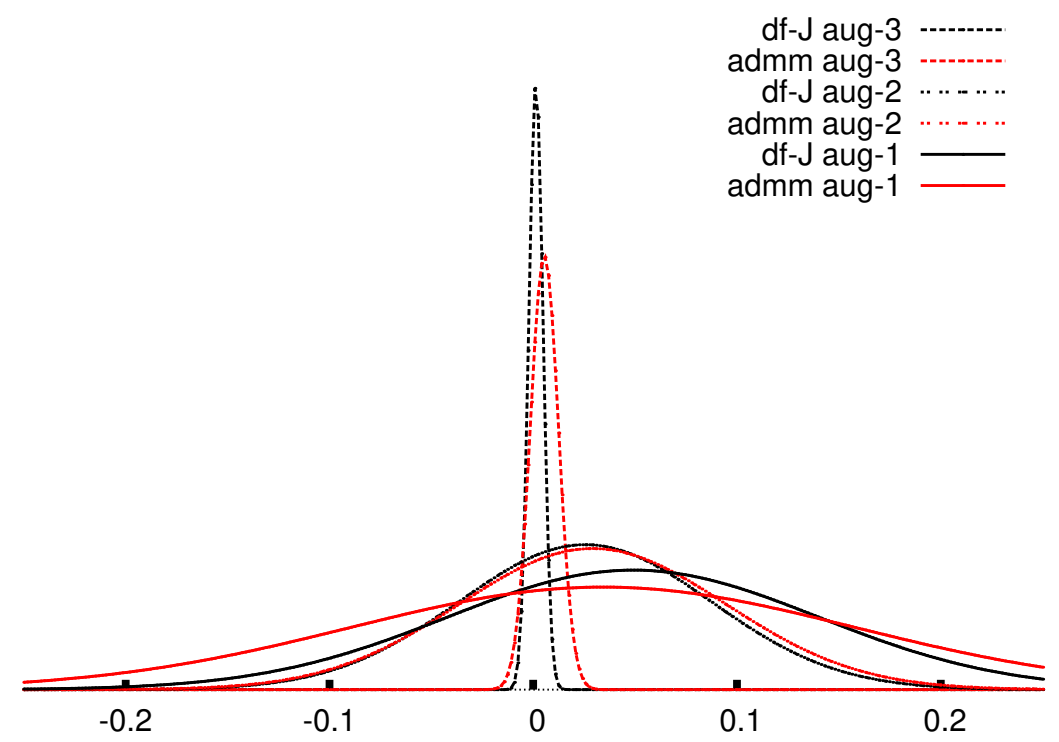

FIGURE 4 The normal distribution of errors in the first five excitation energies (in electron volt) with CAM-B3LYP $(\alpha=0.21, \beta=0.79, \mu=0.45)$ /aug-pcseg- $(n=1,2,3)$ for a set of molecules M11 (see text). Here aug-pcseg-4 results are taken as the reference. 
calculations, respectively. Standard deviations are given in parenthesis. This is already within the range of typical TDDFT excitation errors, of about $0.1 \mathrm{eV}$ or larger (see for instance Ref. [93]). For aug-pcseg- 2 the standard deviations are reduced by another $32-47 \%$, and by roughly an order of magnitude further by going to aug-pcseg-3. See supplementary information Table $\mathrm{S} 2$ for details.

\section{3 | Static polarizabilities}

The normal distribution of the errors in static isotropic polarizabilities at the CAM-B3LYP ( $\alpha=0.21, \beta=0.79$ and $\mu=0.45)$ level for the M11 benchmark is depicted in Figures 5 and 6, for pcseg- $n$ and aug-pcseg- $n$ with $n=1,2,3$, respectively. The isotropic polarizabilities are underestimated in all basis sets and at all levels of theory, with a clear improvement for increasing cardinal number $n$ and upon augmentation. The full numbers are given in Table S3 in the supplementary information. At the reference aug-pcseg-4 full level of theory, the isotropic polarizabilities of the M11 benchmark range from 27.5 to 55.7 a.u. ( 1 a.u. equals $\left.(0.529 \AA \AA)^{3}=0.148 \AA^{3}\right)$. At the pcseg- 1 level the mean errors (standard deviations) are $-6.96(2.70),-6.96(2.70)$ and $-7.32(2.88)$ a.u. for full, $d f-J$ and admm type calculations, respectively, and the errors are reduced by roughly an order of magnitude, to $-0.50(0.20),-0.50(0.20)$ and $-0.84(0.33)$ a.u., at the pcseg- 3 level. Upon augmentation the errors are reduced by one to three orders of magnitude. Already at the aug-pcseg- 1 basis the errors are $-0.31(0.12),-0.31(0.12)$ and $-1.03(0.45)$, whereas at aug-pcseg- 3 they as low as $-3(1),-1(3)$ and $-12(5)$ ma.u. With typical DFT errors ranging from 0.2 to 1.0 a.u., see for instance Ref. [94], basis sets errors are at an acceptable level from the aug-pcseg- 2 level of theory, for all three levels of theory, and remaining basis set errors are essentially removed upon using the aug-pcseg- 3 basis set. The admm type calculations have larger errors in all cases, by up to a factor 4 at the aug-pcseg- 1 level, and for the aug-pcseg- $n$ basis the admm errors more or less bisect the values of the full (and df- $J$ ) calculations of cardinal numbers $n-1$ and $n$.

The error distributions for the static anisotropic polarizabilities are shown in Figures 7 and 8 . For the M11 benchmark the anisotropic polarizabilities range from 8.0 to 47.0 a.u. at the reference aug-pcseg- 4 full level of theory. Although the mean errors are typically slightly smaller than for the isotropic polarizabilities, the standard deviations are larger; as an example the errors are $-0.23(0.35),-0.24(0.36)$ and $-0.38(0.67)$ a.u. for aug-pcseg-1 and $-2(3),-8(9)$ and $-21(25)$ ma.u. for aug-pcseg-3; see Table S4 in the supplementary information for the full list of mean errors and standard deviations. Similar to the isotropic polarizability, the anisotropic polarizabilities are underestimated using aug-pcseg- $n$ basis, with the exception of aug-pcseg-1 admm type calculations. For the pcseg- $n$ basis, the values are instead overestimated. As for the isotropic polarizabilities the admm aug-pcseg- $n$ errors fall in between the aug-pcseg- $(n-1)$ and aug-pcseg- $n$ full errors, although shifted towards the aug-pcseg- $(n-1)$ values, indicating that admm provides a somewhat poorer description of the directional components.

In order to assess the source of the larger ADMM errors in the directional components we chose pyrrole as an example for further investigation. This choice was taken because pyrrole exhibits the largest maximum absolute error in the anisotropic polarizabiltiy for admm/aug-pcseg-1. On inspection three main observations were made: the first 25 excitation energies varied by less than factor two (ranging from 5.74-8.49 eV), the lower state excitation energies converged slightly faster with basis set than the higher ones. However, the excitation energies converged much faster than the transition dipole moments. The results seem to indicate that, not unexpectedly, 1) near degeneracies of the lowest excitation states can enhance errors in the anisotropic polarizabilities for ADMM, and 2) the main source of these errors is connected with the errors in the transition dipole moments. 
df-J 3

admm 3

df-J 2

admm 2

df-J 1

admm 1

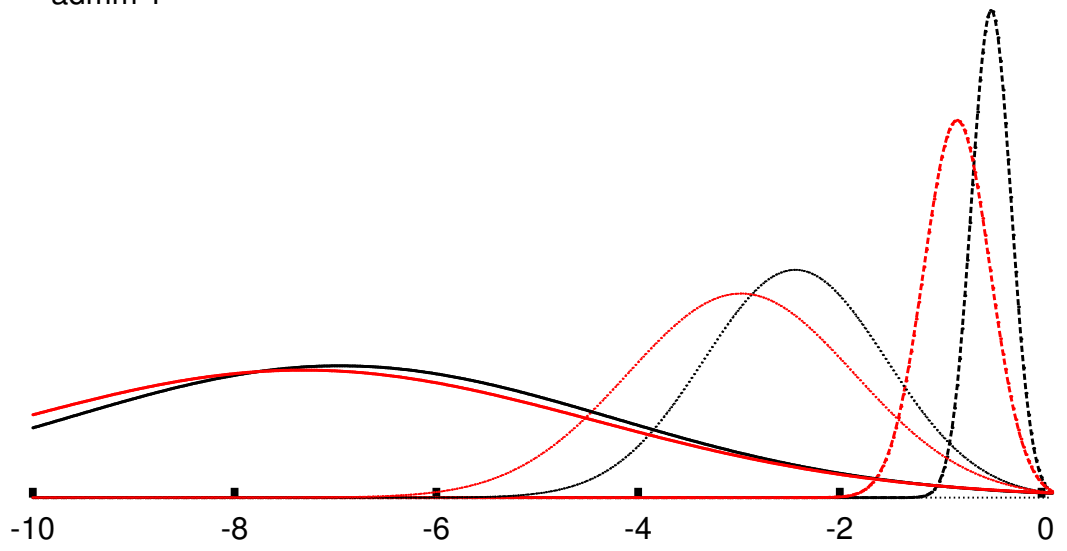

FIGURE 5 The normal distribution of error in the isotropic polarizability (in atomic units) with CAM-B3LYP( $\alpha=0.21, \beta=0.79, \mu=0.45) / p c s e g-n(n=1,2,3)$ for a set of molecules M11 (see text). Here aug-pcseg- 4 results are taken as the reference. 


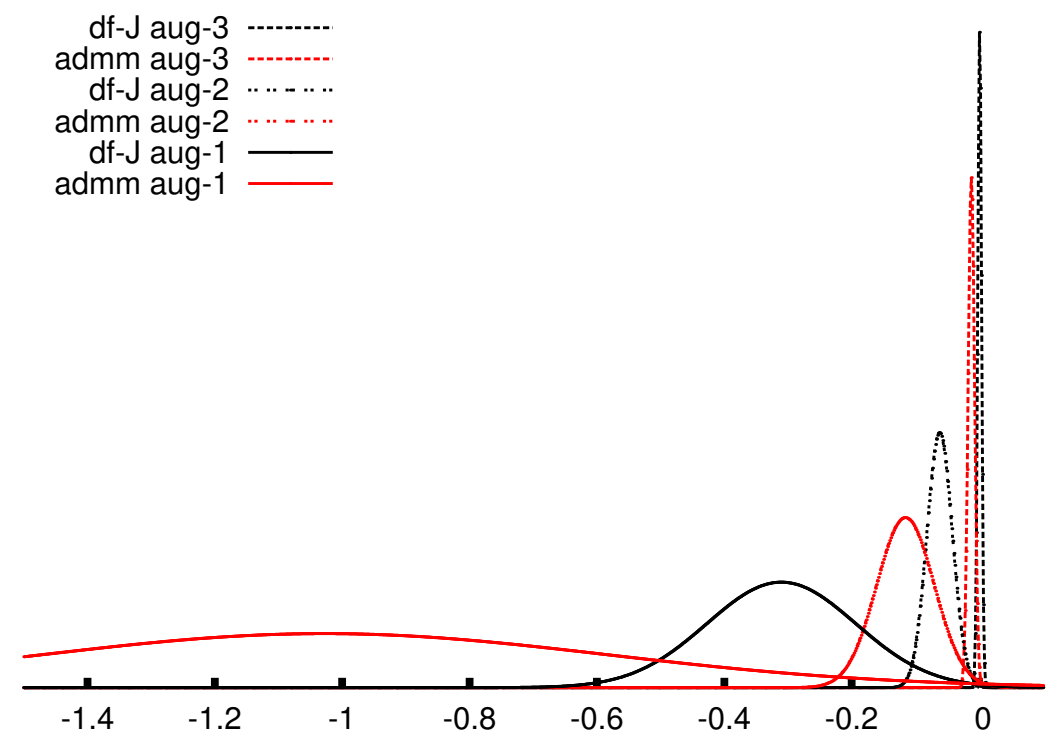

FIGURE 6 The normal distribution of error in the isotropic polarizability (in atomic units) with CAM-B3LYP $(\alpha=0.21, \beta=0.79, \mu=0.45)$ /aug-pcseg- $(n=1,2,3)$ for a set of molecules M11 (see text). Here aug-pcseg-4 results are taken as the reference. 


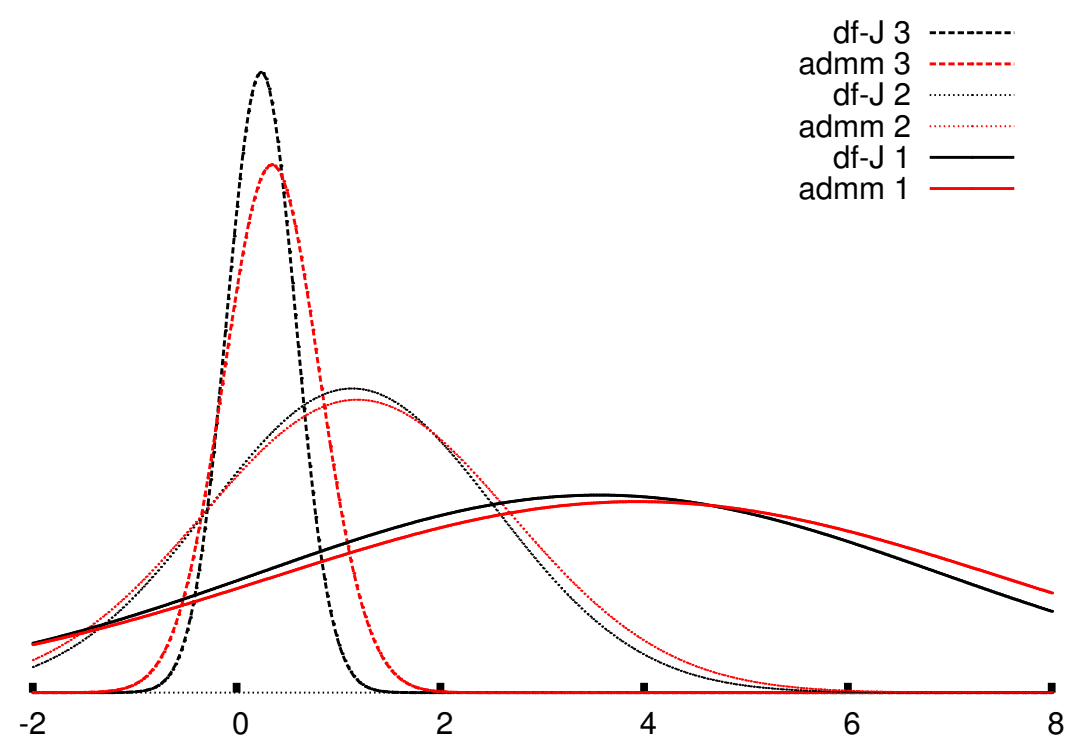

FIGURE 7 The normal distribution of error in the anisotropic polarizability (in atomic units) with CAM-B3LYP $(\alpha=0.21, \beta=0.79, \mu=0.45) / p c s e g-n(n=1,2,3)$ for a set of molecules M11 (see text). Here aug-pcseg-4 results are taken as the reference. 


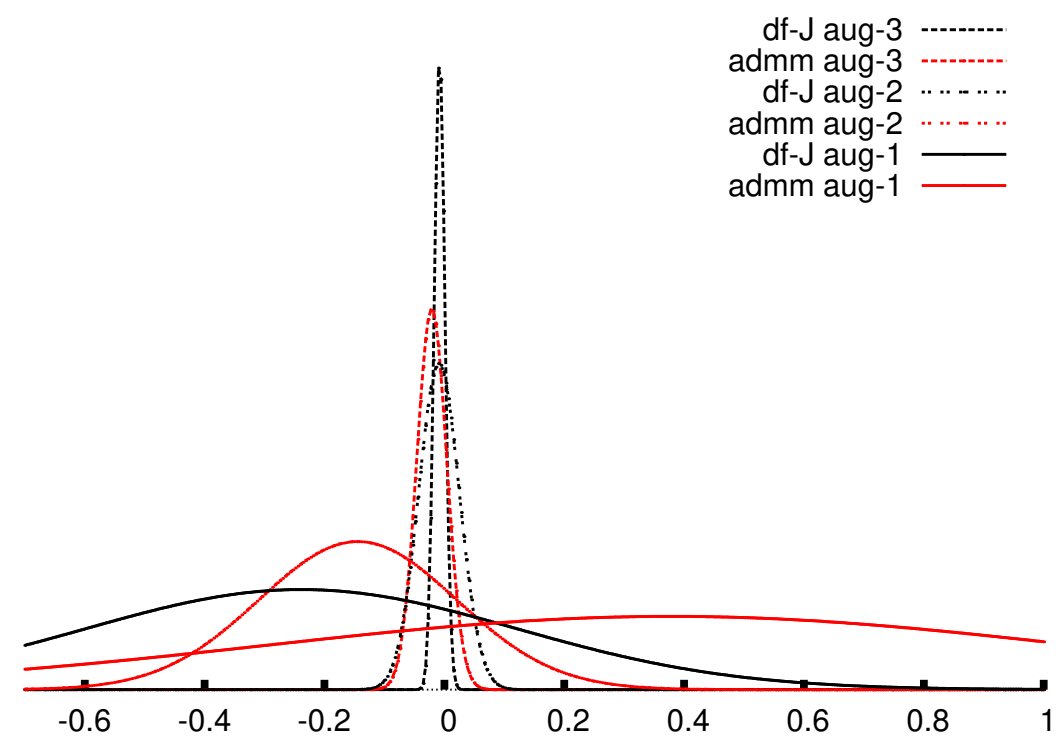

FIGURE 8 The normal distribution of error in the anisotropic polarizability (in atomic units) with CAM-B3LYP $(\alpha=0.21, \beta=0.79, \mu=0.45) /$ aug-pcseg- $n(n=1,2,3)$ for a set of molecules M11 (see text). Here aug-pcseg-4 results are taken as the reference. 


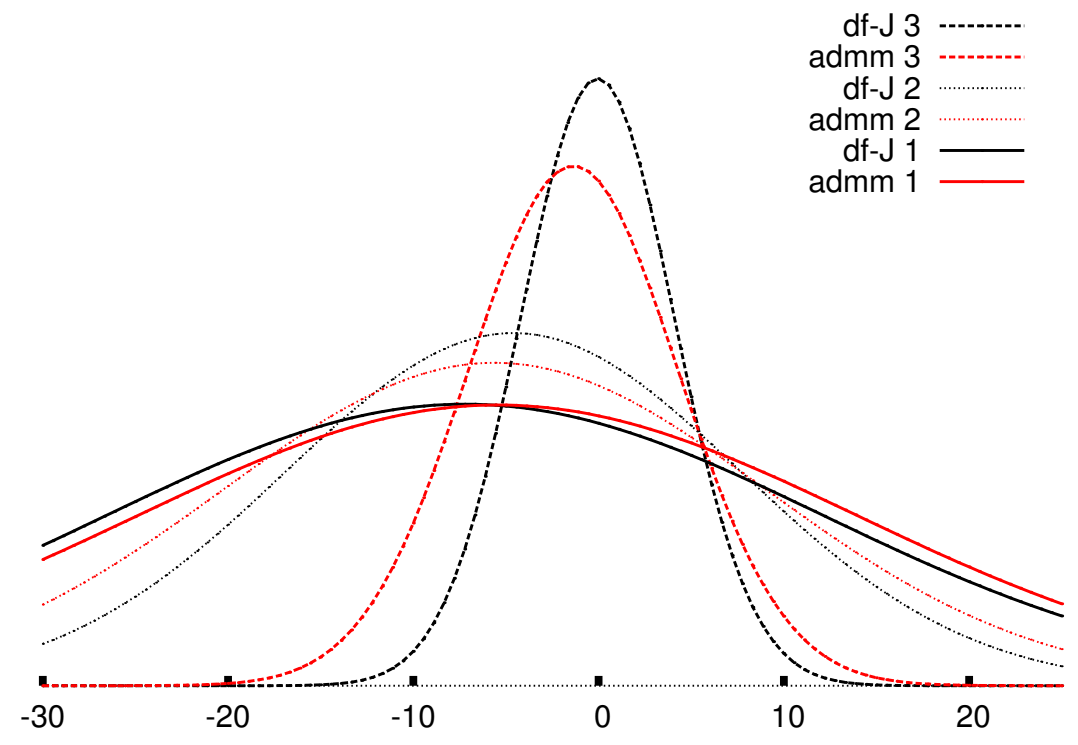

FIGURE 9 The normal distribution of error in the BetaParallel (firsthyperpolarizability) (in atomic units) with CAM-B3LYP $(\alpha=0.21, \beta=0.79, \mu=0.45) / p c s e g-n(n=1,2,3)$ for a set of molecules M11 (see text). Here aug-pcseg- 4 results are taken as the reference.

\section{4 | Static hyperpolarizabilities}

To assess how the methods perform for the first hyperpolarizability we have here chosen to study the component of the dipole first hyperpolarizability tensor $\bar{\beta}$ along the direction of the permanent molecular dipole moment $\boldsymbol{\mu}=\left(\mu_{x}, \mu_{y}, \mu_{z}\right)$, given by $[95,96]$

$$
\bar{\beta}=\frac{3}{5|\mu|}\left(\beta_{x} \mu_{x}+\beta_{y} \mu_{y}+\beta_{z} \mu_{z}\right),
$$

with

$$
\beta_{\xi}=\sum_{\zeta=x, y, z} \beta_{\xi \zeta \zeta}, \quad \xi=x, y, z
$$

where $\beta_{\xi \zeta Y}$ are components of the static first hyperpolarizability tensor. Figures 9 and 10 show the normal distribution of CAM-B3LYP $(\alpha=0.21, \beta=0.79, \mu=0.45)$ errors in the static dipole hyperpolarizability $\bar{\beta}$ for the M11 benchmark, for pcseg- $n$ and aug-pcseg- $n, n=1,2,3$, calculations, respectively, and more detailed results are reported in Tables S5 of the supplementary information.

At the reference aug-pcseg- 4 full calculation the parallel values vary from -41.6 to 15.3 a.u. The standard deviations in the pcseg- $n$ basis are rather large, and vary from 19.3 a.u. for $n=1$ to 4.1 a.u. for $n=3$, for both df- $J$ and full type calculations, and 19.4 to 5.7 a.u. for admm type calculations. Upon augmentation the standard deviations are reduced to the ranges 2.2 to 0.05 a.u. for full, 2.2 to 0.07 a.u. for $\mathrm{df}-J$ and 3.9 to 0.15 for admm type calculations. In all cases the 


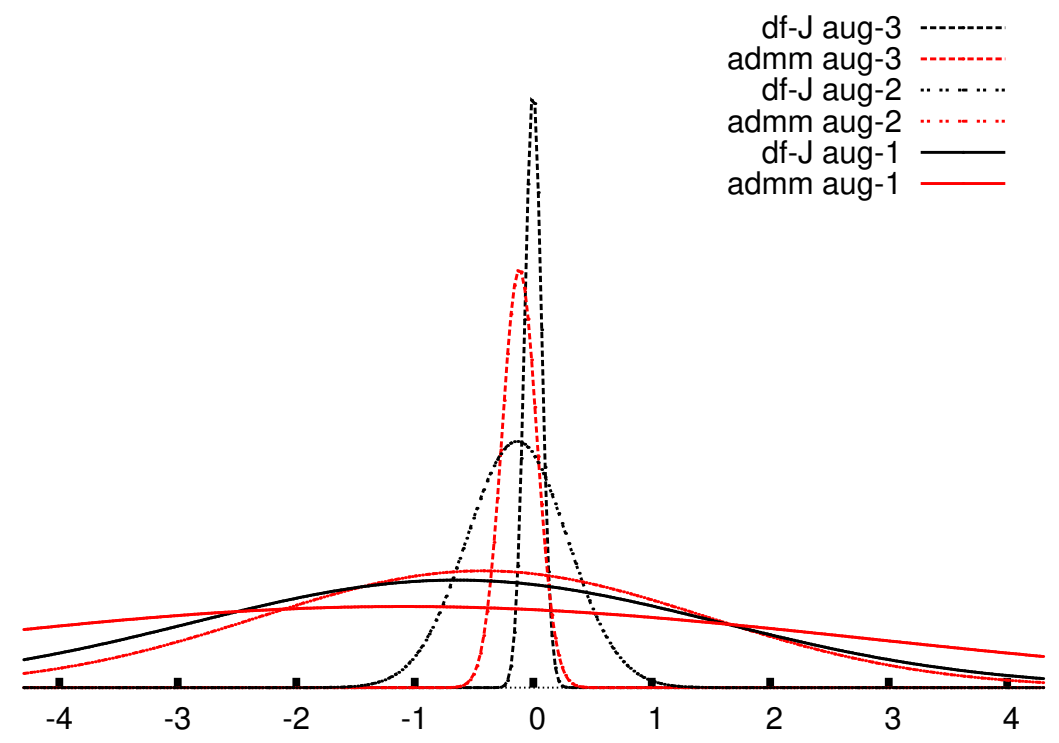

FIGURE 10 The normal distribution of error in the BetaParallel (firsthyperpolarizability) (in atomic units) with CAM-B3LYP $(\alpha=0.21, \beta=0.79, \mu=0.45)$ /aug-pcseg- $(n=1,2,3)$ for a set of molecules M11 (see text). Here aug-pcseg-4 results are taken as the reference. 
TAB LE 1 Average wall timings in seconds per SCF iteration for the acetone molecule, for 'full,' 'df- $J$ ' and 'admm' type calculations (see text for further details).

\begin{tabular}{lccc}
\hline Method type & aug-pcseg-1 & aug-pcseg-2 & aug-pcseg-3 \\
\hline full & 4.1 & 49 & 590 \\
df-J & 3.9 & 43 & 491 \\
admm & 3.2 & 13 & 73 \\
\hline
\end{tabular}

admm- $n$ results falls in between the df- $J-n$ and df- $J-(n-1)$ results; for pcseg- $n$ the admm results are shifted toward $n$, and for aug-pcseg- $n$ the results are less conclusive with the aug-pcseg- 2 admm result close to the aug-pcseg- 1 full value and the aug-pcseg- 3 admm result slightly shifted towards the aug-pcseg- 3 full result.

\section{5 | Performance}

In the previous subsections we have presented results for electronic ground-state energies, the first five excitation energies, polarizabilities and first hyperpolarizabilities for the M11 benchmark set. The results show that density fitting for the Coulomb contribution has negligible effects on the results, and that employing ADMM for the exchange can be done in a systematic fashion, albeit at the cost of reduced accuracy. In this section we give our assessment of performance versus accuracy for acetone as an example molecule, to provide an indicative guide for choosing which method to use in practical calculations.

Averaged timings per SCF iteration for the three different types of calculation (full, df- $J$ and admm) are given in Table 1, for aug-pcseg- $n$ calculations for the acetone molecule, which is on the median of the 11 molecules with regards to computational time. The timings for the response part of the calculation are similar, and we refer the interested reader to the supplementary information Table S6 which includes timings for the individual components for both SCF and the response parts of the calculation. In Table 1 full-type calculations are the sum of reg- $J$, LinK, XC and solver timings. For the df- $J$ entry df- $J$ is used instead of reg- $J$, and the admm entry is the sum of df- $J$, ADMM, XC and solver timings.

The performance gains using density fitting are tremendous when looking at the effect on the Coulomb contribution alone, with speed up factors of 7, 27 and 112 for aug-pcseg- $n$, with cardinal number $n=1,2,3$ respectively. As shown, this performance boost can be attained at little to no loss in accuracy, and density fitting can thus be applied without hesitation. However, for the hybrid DFT calculations presented here, these speed ups have limited effect on the total timings, with only $5 \%, 12 \%$ and $17 \%$ reduction in computational time for the aug-pcseg- $n$ sequence, with $n=1,2,3$ respectively. This is because LinK exchange is the main bottleneck for these calculations, amounting for df- $J$ type calculations to $67 \%, 85 \%$ and $95 \%$ of the total wall time, for $n=1,2,3$.

The motivation to enhance the performance of the exchange contribution should be clear from these results, and the ADMM approximation gives significant speed ups. For the aug-pcseg- $n$ sequence the speed up for the exchange contribution is a factor of 1.5, 5.8 and 9.7, for $n=1,2,3$, respectively. Whilst these speedups are significantly smaller than those obtained for the Coulomb term using density fitting, the combined performance gains using both density fitting for Coulomb and ADMM for exchange are speedups of factors of 1.2, 3.7 and 8.1, respectively. However, these speedups come at a cost of accuracy, mainly due to the ADMM approximation. For electronic ground-state energies, the effect on accuracy due to ADMM is minimal, and the performance gains of ADMM and density fitting outweigh the small loss in accuracy. For excitation energies the same arguments hold, although the speed ups when calculating five 
excitation energies (as done in this paper) are somewhat smaller than for the ground-state energies.

For polarizabilities and hyperpolarizabilities the results are less clear-cut. For the calculation of isotropic polarizabilities ADMM gives a fairly good description still, whereas for the anisotropic polarizabilities this is no longer the case. A possible explanation might be the less satisfactory description of transition dipole contributions that do not effect excitation energies but the polarizability and here in particular the anisotropic one where off diagonal elements might have a stronger weight. For hyperpolarizabilities the admm aug-pcseg-3 results reduce the standard deviation of the full aug-pcseg- 2 results by a factor 2.9 and the additional computational time is only factor 1.5 . Going to the full aug-pcseg-3 the standard deviation is reduced by an additional factor 3.0, now for a factor 8.1 in computational time. Clearly the admm aug-pcseg-3 type calculation is a valid and efficient option. This does not hold for admm aug-pcseg-2 however. Going from aug-pcseg-1 full type calculations reduce the standard deviation by factor 1.2 for an increase in computational time by factor 3.2. Using instead the full aug-pcseg- 2 reduces the error by factor 4.5 for a cost of factor 3.7 in calculation time.

In summary, the presented results indicate that density fitting of the Coulomb contribution can be used without hesitation for all properties studied here, although the overall performance gains for hybrid functionals are limited by the efficiency of the exchange contribution. The ADMM exchange approximation can readily be applied for ground-state and excitation energies, whereas for polarizabilities and hyperpolarizabilities the performance gains come at a larger cost in accuracy, making the method of choice less definite.

\section{6 | P700 tetramer model system}

Recently, Suomivuori et. al. [74] published a computational study on the lowest vertical excitation energies of a model system of the special cholorphyll/bacteriochlorphyll pigment center P700 of photosystem I, where the label P700 refers to the absorption peak at $700 \mathrm{~nm}$. The $\mathrm{P} 700$ tetramer model is shown in Figure 11. It consists of four chlorin molecules ligated with histidine residues and water. In their study Suomivuori et. al. [74] used the algebraic diagrammatic construction through second order $\operatorname{ADC}(2)$ approach [97, 98], providing highly accurate ab-initio data for a multimeric compound. The P700 tetramer model consists of 198 atoms and serves in the present work as a test system for assessing the accuracy of the different approximative methods applied in our work. It provides also an example for the potential applicability of the LSDalton response module [86] for biologically relevant molecules.

In Table 2 calculated CAM-B3LYP ( $\alpha=0.21, \beta=0.79, \mu=0.45)$ results are reported for pcseg-1 and pcseg- 2 and compared to those of Ref. [74]. The five lowest vertical transitions have been examined, and the results of augmentation are included at the admm level of theory for aug-pcseg-1 and aug-pcseg-2. The results obtained without any approximation (full) and with density-fitting (df- $J$ ) are very similar as compared to the ones of the combined density-fitting and ADMM approach (admm). An increase of the basis set from pcseg-1 to aug-pcseg-2 leads only to minor changes in the excitation energies, which are slightly decreased by about $0.1 \mathrm{eV}$ or less. The obtained results suggest that augmentation of the basis is not needed for the present system. It appears to be sufficient to use a pcseg- 2 quality basis set or even pcseg-1. The calculated CAM-B3LYP $(\alpha=0.21, \beta=0.79, \mu=0.45)$ results agree well with the published ADC(2) reference values, which have been obtained using the def2-TZVP basis [18]. The def2-TZVP basis is of similar quality as the pcseg-2 basis used in the present work. The experimental value for the lowest transition of the full P700 system lies at $1.77 \mathrm{eV}[99,100,101,102]$. On ADC(2) level the lowest excitation energy lies at $2.02 \mathrm{eV}$, while we obtain $1.88 \mathrm{eV}$ for CAM-B3LYP( $\alpha=0.21, \beta=0.79, \mu=0.45)$ with admm/aug-pcseg- 2 .

For the sake of completeness we also tested different DFT functionals for the present P700 model system. The results for BLYP [103, 104], B3LYP [105, 104], CAM-B3LYP and CAM-B3LYP ( $\alpha=0.21, \beta=0.79$ and $\mu=0.45)$ are summarized in Table 3, where CAM-B3LYP is the standard version, with the functional parameters $\alpha=0.19, \beta=0.46$ and 


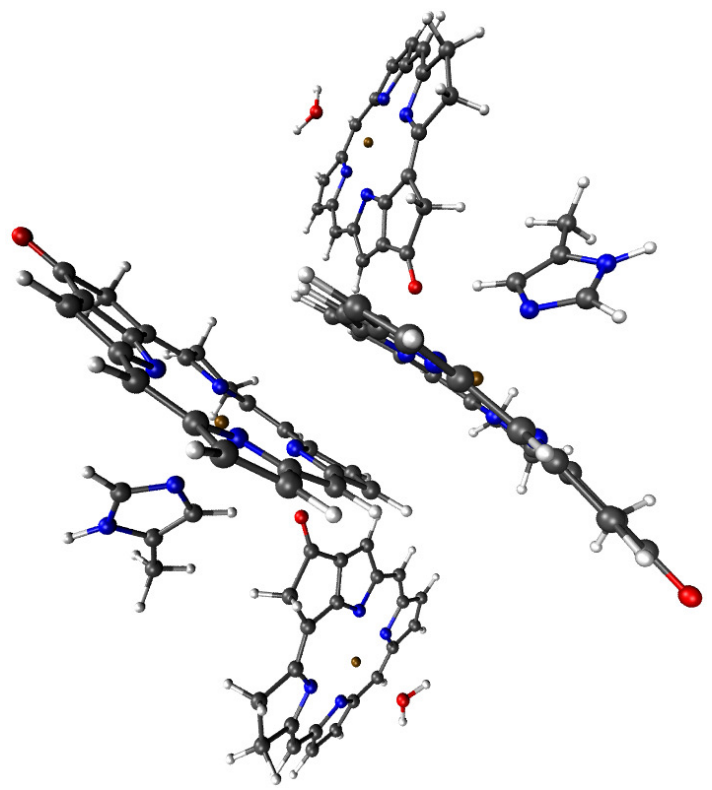

FIGURE 11 P700 -tetramer model system. The coordinates are taken from Ref. [74]

$\mu=0.33$. In this sequence of DFT functionals, the long-range exchange proportion varies from zero to one, starting from no exchange for BLYP, followed by 0.2 for B3LYP, 0.65 for CAM-B3LYP and full inclusion for CAM-B3LYP $(\alpha=0.21$, $\beta=0.79$ and $\mu=0.45$ ). Neither the BLYP or the B3LYP functionals describe the P700 tetramer well, and underestimated the excitation energies by roughly $1.4 \mathrm{eV}$ and $0.8 \mathrm{eV}$ as compared to Ref. [74], respectively. In contrast, the excitation energies obtained with both flavors of the CAM-B3LYP functional perform very well. This can be explained by the improved description of the long-range exchange.

The total computational cost for the pcseg- 2 calculations of the five lowest excitation energies using 1024 cores, were 7.4 hours for admm ( $1.3 \mathrm{~h} \mathrm{SCF}+6.1 \mathrm{~h}$ response), 36.2 hours for $\mathrm{df}-J(11.5 \mathrm{~h} \mathrm{SCF}+24.7 \mathrm{~h}$ response), and 42.5 hours for the full calculation ( $14.2 \mathrm{~h} \mathrm{SCF}+28.3 \mathrm{~h}$ response).

\section{6 | SUMMARY AND CONCLUSIONS}

We have presented an extension to the standard formulation of response theory, which accounts for approximate Fock/Kohn-Sham matrices such as the matrices used in density fitting or the ADMM2 approximation. The development represents a framework to easily introduce the approximate methodologies used in ground-state electronic structure calculations to Hartree-Fock/Kohn-Sham response theory, provided that the Coulomb and exchange matrices can be formulated as derivatives of the approximated Coulomb and Exchange energies.

The option for the combined use of density fitting and ADMM has been implemented and tested in the LSDalton program [86]. An error analysis with respect to different basis sets for full, $\mathrm{df}-J$ and admm type calculations has been performed for DFT ground state energies, vertical excitation energies, static polarizabilities and hyperpolarizabilities for a set of 11 small to medium sized molecules, against reference aug-pcseg- 4 results. 
TAB LE 2 Calculated CAM-B3LYP $(\alpha=0.21, \beta=0.79$ and $\mu=0.45)$ excitation energies in eV for the P700 tetramer model using the pcseg- 1 and pcseg- 2 basis sets. Results obtained for the aug-pcseg- 1 and aug-pcseg- 2 basis sets with admm are given in parenthesis.

\begin{tabular}{cccccccc}
\hline \multirow{2}{*}{ state } & \multicolumn{3}{c}{ pcseg-1 } & \multicolumn{3}{c}{ pcseg-2 } \\
& full & df-J & admm & full & df-J & admm & Ref. [74] \\
\hline 1 & 1.95 & 1.95 & $1.98(1.94)$ & 1.89 & 1.89 & $1.90(1.88)$ & 2.02 \\
\hline 2 & 2.00 & 2.00 & $2.04(1.93)$ & 1.94 & 1.94 & $1.95(1.93)$ & 2.04 \\
\hline 3 & 2.01 & 2.01 & $2.05(2.01)$ & 1.95 & 1.95 & $1.97(1.94)$ & 2.05 \\
\hline 4 & 2.04 & 2.04 & $2.08(2.04)$ & 1.98 & 1.98 & $2.00(1.98)$ & 2.06 \\
\hline 5 & 2.61 & 2.61 & $2.57(2.56)$ & 2.58 & 2.58 & $2.59(2.58)$ & \\
\hline
\end{tabular}

TA B LE 3 Calculated excitation energies in $\mathrm{VV}$ for the P700 tetramer model using different functionals and admm approximation. For all calculations the pcseg- 2 basis has been used. The CAM-B3LYP results labeled with * are obtained with the specifications $\alpha=0.21, \beta=0.79$ and $\mu=0.45$, rather than the standard CAM-B3LYP functional parameters $\alpha=0.19, \beta=0.46$ and $\mu=0.33$.

\begin{tabular}{cccccc}
\hline state & BLYP & B3LYP & CAM-B3LYP & CAM-B3LYP* & Ref. [74] \\
\hline 1 & 0.63 & 1.22 & 2.06 & 1.90 & 2.02 \\
2 & 0.64 & 1.23 & 2.10 & 1.95 & 2.04 \\
3 & 0.68 & 1.25 & 2.12 & 1.97 & 2.05 \\
4 & 0.69 & 1.27 & 2.14 & 2.00 & 2.06 \\
5 & 0.76 & 1.38 & 2.31 & 2.59 & \\
\hline \hline
\end{tabular}


The presented results indicate that density fitting of the Coulomb contribution can be used without hesitation for all properties studied here, although the overall performance gains for hybrid functionals are limited due to the high computational cost of the exchange contribution. The ADMM exchange approximation can be applied to accelerate the evaluation of the exchange contribution, although this entails introducing additional approximations. The ADMM approach was found to work well for ground-state and excitation energies, making it the method of choice for these properties. For polarizabilities and hyperpolarizabilities, although the accuracy with basis set size still improves systematically, the performance gains come at a higher cost in accuracy - making the method of choice less definite.

The lowest five vertical singlet excitation energies for a tetrameric model system of the P700 special pigment [74] of photosystem I have been studied, demonstrating the applicability of the LSDalton response module [86] for a large molecule of biological relevance. Different functionals and basis sets have been tested, and our results indicate that augmentation of the basis is not needed for this system. Satisfactory results can be obtained already on pcseg-1 level, as compared to previously published ADC(2) benchmark data [74], using our combined density-fitting/ADMM approach with the CAM-B3LYP functional.

\section{7 | SUPPLEMENTARY INFORMATION}

See supplementary information for detailed results.

\section{ACKNOWLEDGMENT}

The research leading to these results has received funding from the European Research Council under the European Union's Seventh Framework Programme (FP/2007-2013)/ERC Grant Agreement no. 291371. CK, SR and HF thank the Norwegian Research Council through the CoE Hylleraas Centre for Quantum Molecular Sciences (Grant No. 262695 and 231571/F20) for support. This work has received support from the Norwegian Supercomputing Program(NOTUR) through a grant of computer time (Grant No. NN4654K). A. M. T. gratefully acknowledges support from the Royal Society University Research Fellowship scheme the Engineering and Physical Sciences Research Council (EPSRC), (Grant No. EP/M029131/1). 


\section{9 | APPENDIX}

\section{1 | Time dependent Schrödinger equation matrix elements}

In the time-dependent Schrödinger equation of Eq. (5) the Kohn-Sham matrix F is defined through

$$
F_{\mu \nu}(\mathrm{D})=\frac{\delta E}{\delta D_{\mu \nu}}=h_{\mu \nu}+J_{\mu \nu}+w K_{\mu \nu}+K_{\mu \nu}^{\mathrm{xc}}
$$

where

$$
\begin{aligned}
h_{\mu \nu} & =\int \chi_{\mu}(\mathbf{r})\left(-\frac{1}{2} \nabla_{I}^{2}+\sum_{I} \frac{Z_{I}}{\left|\mathbf{r}-\mathbf{R}_{I}\right|}\right) \chi_{v}(\mathbf{r}) d \mathbf{r} \\
J_{\mu \nu}(\mathbf{D}) & =\sum_{\rho \sigma}(\mu \nu \mid \rho \sigma) D_{\rho \sigma} \\
K_{\mu \nu}(\mathbf{D}) & =\sum_{\rho \sigma}(\mu \sigma \mid \rho v) D_{\rho \sigma} \\
F_{\mu \nu}^{\mathrm{xC}}(\mathbf{D}) & =\int \chi_{\mu}(\mathbf{r}) v_{\mathrm{xc}}(\mathbf{r}, t) \chi_{\nu}(\mathbf{r}) d \mathbf{r}
\end{aligned}
$$

with the two-electron integrals in Mulliken form

$$
(\mu v \mid \rho \sigma)=\iint \chi_{\mu}\left(\mathbf{r}_{1}\right) \chi_{v}\left(\mathbf{r}_{1}, t\right) \frac{1}{r_{12}} \chi_{\rho}\left(\mathbf{r}_{2}\right) \chi_{\sigma}\left(\mathbf{r}_{2}\right) d \mathbf{r}_{1} d \mathbf{r}_{2}
$$

The matrix elements of the time-dependent perturbation operator are given by

$$
V_{\mu \nu}=\int \chi_{\mu}(\mathbf{r}) V(\mathbf{r}, t) \chi_{\nu}(\mathbf{r}) d \mathbf{r}
$$

and the overlap matrix elements are given in the usual form

$$
S_{\mu \nu}=\int \chi_{\mu}(\mathbf{r}) \chi_{\nu}(\mathbf{r}) d \mathbf{r}
$$

\section{2 | Kohn-Sham Fock matrix expansion terms}

The components of the matrix $\mathrm{G}(\mathrm{M})$ in Equation (10) are given by

$$
\begin{aligned}
J_{\mu v}(\mathrm{M}) & =\sum_{\rho \sigma}(\mu v \mid \rho \sigma) M_{\rho \sigma} \\
K_{\mu \nu}(\mathrm{M}) & =\sum_{\rho \sigma}(\mu \sigma \mid \rho v) M_{\rho \sigma} \\
K_{\mu \nu}^{\mathrm{xc}}(\mathbf{M}) & =\iint \chi_{\mu}(\mathbf{r}) \sum_{\rho \sigma} \frac{\delta v_{\mathrm{xc}}}{\delta \rho(\mathbf{r}, t)} \chi_{\rho}(\mathbf{r}) \chi_{\sigma}(\mathbf{r}) \mathbf{M}_{\rho \sigma} \chi_{\nu}(\mathbf{r}) d \mathbf{r} d \mathbf{r}^{\prime},
\end{aligned}
$$

where the Coulomb $\mathrm{J}(\mathrm{M})$ and exchange $\mathrm{K}(\mathrm{M})$ matrices have the same form as in the KS matrix of Eq. (46), whereas the exchange-correlation contribution $\mathrm{K}^{\mathrm{Xc}}(\mathrm{M})$ is more involved than the corresponding exchange-correlation contribution 
$\mathrm{F}^{\mathrm{xC}}(\mathrm{D})$ to the KS matrix.

In the equation (11), the term $T^{\mathrm{xc}}(\mathrm{N}, \mathrm{M})$ is given by

$$
\begin{array}{r}
T_{\phi \xi}^{\mathrm{xc}}(\mathbf{N}, \mathbf{M})=\sum_{\rho \sigma \eta \epsilon} M_{\rho \sigma} N_{\eta \epsilon} \iiint \chi_{\phi}^{*}(\mathbf{r}) \chi_{\xi}(\mathbf{r}) \chi_{\rho}^{*}\left(\mathbf{r}^{\prime}\right) \\
\chi_{\sigma}\left(\mathbf{r}^{\prime}\right) \chi_{\eta}^{*}\left(\mathbf{r}^{\prime \prime}\right) \chi_{\epsilon}\left(\mathbf{r}^{\prime \prime}\right) \frac{\delta^{2} v_{\mathrm{xc}}(\mathbf{r})}{\delta \rho(\mathbf{r})^{2}} \mathrm{~d} \mathbf{r} \mathbf{d} \mathbf{r}^{\prime} \mathbf{d} \mathbf{r}^{\prime \prime} .
\end{array}
$$

\section{3 | Quadratic Response Equation}

The terms contributing to the linear response equation has been shown in Section 2.2. Here we discuss the terms that contribute to the quadratic response equation.

$$
\begin{aligned}
& -\left(\mathbf{G}\left(\left[\mathbf{D}, \mathbf{X}^{(2)}\left(\omega_{1}, \omega_{2}\right)\right]_{\mathbf{S}}\right)\right. \\
& +P_{12} \mathbf{G}\left(\frac{1}{2}\left[\left[\mathbf{D}, \mathbf{X}^{(1)}\left(\omega_{1}\right)\right], \mathbf{X}^{(2)}\left(\omega_{2}\right)\right]\right) \\
& \left.+\frac{1}{2} P_{12} \mathbf{T}\left(\left[\mathbf{D}, \mathbf{X}^{(1)}\left(\omega_{1}\right)\right]_{\mathbf{S}},\left[\mathbf{D}, \mathbf{X}^{(1)}\left(\omega_{2}\right)\right]_{\mathbf{S}}\right)\right) \mathbf{D S} \\
& +\mathbf{S D}\left(\mathbf{G}\left(\left[\mathbf{D}, \mathbf{X}^{(2)}\left(\omega_{1}, \omega_{2}\right)\right]_{\mathbf{S}}\right)\right. \\
& +P_{12} \mathbf{G}\left(\frac{1}{2}\left[\left[\mathbf{D}, \mathbf{X}^{(1)}\left(\omega_{1}\right)\right], \mathbf{X}^{(2)}\left(\omega_{2}\right)\right]\right) \\
& \left.+\frac{1}{2} P_{12} \mathbf{T}\left(\left[\mathbf{D}, \mathbf{X}^{(1)}\left(\omega_{1}\right)\right]_{\mathbf{S}},\left[\mathbf{D}, \mathbf{X}^{(1)}\left(\omega_{2}\right)\right]_{\mathbf{S}}\right)\right) \\
& -P_{12} \mathbf{G}\left(\left[\mathbf{D}, \mathbf{X}^{(1)}\left(\omega_{1}\right)\right]_{\mathbf{S}}\right)\left[\mathbf{D}, \mathbf{X}^{(1)}\left(\omega_{2}\right)\right]_{\mathbf{S}} \mathbf{S} \\
& -\frac{1}{2} P_{12} \mathbf{F}\left(\mathbf{D}_{0}\right)\left[\left[\mathbf{D}, \mathbf{X}^{(1)}\left(\omega_{1}\right)\right], \mathbf{X}^{(1)}\left(\omega_{2}\right)\right] \mathbf{S} \\
& +P_{12} \mathbf{S}\left[\mathbf{D}, \mathbf{X}^{(1)}\left(\omega_{1}\right)\right]_{\mathbf{S}} \mathbf{G}\left(\left[\mathbf{D}, \mathbf{X}^{(1)}\left(\omega_{2}\right)\right]_{\mathbf{S}}\right) \\
& +\frac{1}{2} P_{12} \mathbf{S}\left[\left[\mathbf{D}, \mathbf{X}^{(1)}\left(\omega_{1}\right)\right], \mathbf{X}^{(1)}\left(\omega_{2}\right)\right] \mathbf{F}\left(\mathbf{D}_{0}\right) \\
& -\mathbf{F}\left[\mathbf{D}, \mathbf{X}^{(2)}\left(\omega_{1}, \omega_{2}\right)\right]_{\mathbf{S}} \mathbf{S}+\mathbf{S}\left[\mathbf{D}, \mathbf{X}^{(2)}\left(\omega_{1}, \omega_{2}\right)\right]_{\mathbf{S}} \mathbf{F} \\
& -\left(\omega_{1}+\omega_{2}\right) \mathbf{S}\left[\mathbf{D}, \mathbf{X}^{(2)}\left(\omega_{1}, \omega_{2}\right)\right]_{\mathbf{S}} \mathbf{S} \\
& -\frac{1}{2}\left(\omega_{1}+\omega_{2}\right) P_{12} \mathbf{S}\left[\left[\mathbf{D}, \mathbf{X}^{(1)}\left(\omega_{1}\right)\right], \mathbf{X}^{(2)}\left(\omega_{2}\right)\right] \mathbf{S} \\
& =\mathbf{V}\left[\mathbf{D}, \mathbf{X}^{(2)}\left(\omega_{1}, \omega_{2}\right)\right]_{\mathbf{S}} \mathbf{S}-\mathbf{S}\left[\mathbf{D}, \mathbf{X}^{(2)}\left(\omega_{1}, \omega_{2}\right)\right]_{\mathbf{S}} \mathbf{V}
\end{aligned}
$$

To obtain the expressions above we have used the Fourier expansion of $X^{(1)}(t)$ (see Eq. (14)) and $X^{(2)}(t)$

$$
X^{(2)}(t)=\iint_{-\infty}^{\infty} \exp \left(-i\left(\omega_{1}+\omega_{1}\right) t\right) X^{(2)}\left(\omega_{1}, \omega_{2}\right) d \omega
$$

Furthermore, we require the second order correction to be symmetric in the frequencies. The integration variables $\omega_{1}$ and $\omega_{2}$ have been symmetrized using the operator $P_{12}$, which creates the different permutations of the frequencies $\omega_{1}$ 
and $\omega_{2}$. Finally, using the definitions in Eq. (17) and (18) along with

$$
\begin{aligned}
& \mathbf{E}^{[3]} \mathbf{X}^{(1)}\left(\omega_{1}\right) \mathbf{X}^{(1)}\left(\omega_{2}\right)= \\
& -\left(\mathbf{G}\left(\frac{1}{2}\left[\left[\mathbf{D}, \mathbf{X}^{(1)}\left(\omega_{1}\right)\right], \mathbf{X}^{(2)}\left(\omega_{2}\right)\right]\right)\right. \\
& \left.\quad+\frac{1}{2} \mathbf{T}\left(\left[\mathbf{D}, \mathbf{X}^{(1)}\left(\omega_{1}\right)\right]_{\mathbf{S}},\left[\mathbf{D}, \mathbf{X}^{(1)}\left(\omega_{2}\right)\right]_{\mathbf{S}}\right)\right) \mathbf{D S} \\
& +\mathbf{S D}\left(\mathbf{G}\left(\frac{1}{2}\left[\left[\mathbf{D}, \mathbf{X}^{(1)}\left(\omega_{1}\right)\right], \mathbf{X}^{(2)}\left(\omega_{2}\right)\right]\right)\right. \\
& \left.\quad+\frac{1}{2} \mathbf{T}\left(\left[\mathbf{D}, \mathbf{X}^{(1)}\left(\omega_{1}\right)\right]_{\mathbf{S}},\left[\mathbf{D}, \mathbf{X}^{(1)}\left(\omega_{2}\right)\right]_{\mathbf{S}}\right)\right) \\
& -\mathbf{G}\left(\left[\mathbf{D}, \mathbf{X}^{(1)}\left(\omega_{1}\right)\right]_{\mathbf{S}}\right)\left[\mathbf{D}, \mathbf{X}^{(1)}\left(\omega_{2}\right)\right]_{\mathbf{S}} \mathbf{S} \\
& -\frac{1}{2} \mathbf{F}\left(\mathbf{D}_{0}\right)\left[\left[\mathbf{D}, \mathbf{X}^{(1)}\left(\omega_{1}\right)\right], \mathbf{X}^{(1)}\left(\omega_{2}\right)\right] \mathbf{S} \\
& +\mathbf{S}\left[\mathbf{D}, \mathbf{X}^{(1)}\left(\omega_{1}\right)\right]_{\mathbf{S}} \mathbf{G}\left(\left[\mathbf{D}, \mathbf{X}^{(1)}\left(\omega_{2}\right)\right]_{\mathbf{S}}\right) \\
& +\frac{1}{2} \mathbf{S}\left[\left[\mathbf{D}, \mathbf{X}^{(1)}\left(\omega_{1}\right)\right], \mathbf{X}^{(1)}\left(\omega_{2}\right)\right] \mathbf{F}\left(\mathbf{D}_{0}\right) \mathbf{S}^{[3]} \mathbf{X}^{(1)}\left(\omega_{1}\right) \mathbf{X}^{(1)}\left(\omega_{2}\right)
\end{aligned}
$$

and,

$$
\mathbf{S}^{[3]} \mathbf{X}^{(1)}\left(\omega_{1}\right) \mathbf{X}^{(1)}\left(\omega_{2}\right)=\frac{1}{2} \mathbf{S}\left[\left[\mathbf{D}, \mathbf{X}^{(1)}\left(\omega_{1}\right)\right], \mathbf{X}^{(2)}\left(\omega_{2}\right)\right] \mathbf{S}
$$

we obtain the well known form of the quadratic response equation of Eq. (20).

\section{4 | The CAM-B3LYP correction functional}

The DFT functional CAM-B3LYP [106], is based on a trivial splitting of the Coulomb operator into a short- and a long-range part, according to

$$
\frac{1}{r_{12}}=\frac{1-\alpha-\beta \operatorname{erf}\left(\mu r_{12}\right)}{r_{12}}+\frac{\alpha+\beta \operatorname{erf}\left(\mu r_{12}\right)}{r_{12}}
$$

where the choice of parameters $\alpha, \beta$ and $\mu$ specify the splitting. The error function, $\operatorname{erf}\left(\mu r_{12}\right)$, is a smooth function in the range [0 -1$]$, starting at zero and approching unity for large distances. The short-range part is evaluated using DFT

$$
E_{\mathrm{X}}^{\mathrm{CAM}}=(1-\alpha)\left(E_{\mathrm{X}}^{\mathrm{Dirac}}+\Delta E_{\mathrm{X}}^{\mathrm{B} 88}\right)-\beta E_{\mathrm{X}}^{\mathrm{Sr}},
$$

with $E_{\mathrm{x}}^{\mathrm{Dirac}}$ and $\Delta E_{\mathrm{x}}^{\mathrm{B} 88}$ the Dirac [107] and Becke [103] exchange functionals, and $E_{\mathrm{x}}^{\mathrm{sr}}$ the short-range exchange functional of likura et al. [108]. The long-range part is evaluated as a range-separated HF exchange contribution

$$
E_{\mathrm{k}}^{\mathrm{CAM}}=\frac{1}{2} \sum_{a b c d} D_{a c}\left(a b\left|\frac{\alpha+\beta \operatorname{erf}\left(\mu r_{12}\right)}{r_{12}}\right| c d\right) D_{b d} .
$$

In addition to the short- and long-range contributions, CAM-B3LYP also includes correlation in the same way as B3LYP, namely 0.19 of the VWN5 [109] and 0.81 of the LYP [104] functionals. For ADMM we have constructed the 
complementary GGA functional

$$
E_{\mathrm{x}}^{\mathrm{COMP}}=\alpha\left(E_{\mathrm{x}}^{\mathrm{Dirac}}+\Delta E_{\mathrm{x}}^{\mathrm{B} 88}\right)+\beta E_{\mathrm{x}}^{\mathrm{sr}}
$$

to approximate the exchange term, Eq. (58), which we employ for the two correction terms $E_{\mathrm{admm}}^{\mathrm{x}}[\rho]$ and $E_{\mathrm{admm}}^{\mathrm{x}}\left[\rho_{\mathrm{admm}}\right]$ in Eq. (33) when using the CAM-B3LYP DFT functional.

\section{REFERENCES}

[1] Simen Reine, Trygve Helgaker, and Roland Lindh. Multi-electron integrals. Wiley Interdisciplinary Reviews: Computational Molecular Science, 2(2):290-303, 2012.

[2] J. L. Whitten. Coulombic potential energy integrals and approximations. J. Chem. Phys., 58:4496-4501, 1973.

[3] J A Jafri and J L Whitten. Electron repulsion integral approximations and error bounds: Molecular applications. J. Chem. Phys., 61:2116-2121, 1974.

[4] E. J. Baerends, D. E. Ellis, and P. Ros. Self-consistent molecular Hartree-Fock-Slater calculations. I. The computational procedure. Chem. Phys., 2:41-51, 1973.

[5] B. I. Dunlap, J. W. D. Connolly, and J. R. Sabin. On some approximations in applications of $X_{\alpha}$ theory. J. Chem. Phys., 71: 3396-3402, 1979.

[6] B. I. Dunlap, J. W. D. Connolly, and J. R. Sabin. On first-row diatomic molecules and local density models. J. Chem. Phys., 71:4993-4999, 1979.

[7] O. Vahtras, J. Almlöf, and M. W. Feyereisen. Integral approximations for LCAO-SCF calculations. Chem. Phys. Lett., 213: 514-518, 1993.

[8] Frank Neese. An Improvement of the Resolution of the Identity Approximation for the Formation of the Coulomb Matrix. J. Comput. Chem., 24(14):1740-1747, 2003.

[9] Simen Reine, Andreas Krapp, Maria Francesca lozzi, Vebjørn Bakken, Trygve Helgaker, Filip Pawłowski, and Pawel Sałek. An efficient density-functional-theory force evaluation for large molecular systems. J. Chem. Phys., 133:044102, 2010.

[10] F Weigend. A fully direct RI-HF algorithm: Implementation, optimised auxiliary basis sets, demonstration of accuracy and efficiency. Phys. Chem. Chem. Phys., 4:4285-4291, 2002.

[11] Robert Polly, Hans-Joachim Werner, Frederick R. Manby, and Peter J. Knowles. Fast Hartree-Fock theory using local density fitting approximations. Mol. Phys., 102:2311-2321, 2004.

[12] Alex Sodt, Joseph E Subotnik, and Martin Head-Gordon. Linear scaling density fitting. J. Chem. Phys., 125:194109, 2006.

[13] A Sodt and M Head-Gordon. Hartree-Fock exchange computed using the atomic resolution of the identity approximation. J. Chem. Phys., 128:104106, 2008.

[14] Simen Reine, Erik Tellgren, Andreas Krapp, Thomas Kjærgaard, Trygve Helgaker, Branislav Jansík, Stinne Høst, and Paweł Sałek. Variational and robust density fitting of four-center two-electron integrals in local metrics. J. Chem. Phys., 129:104101, 2008.

[15] B I Dunlap. Robust variational fitting: Gaspar's variational exchange can accurately be treated analytically. J. Mol. Struct. (Theochem), 501:221-228, 2000. 
[16] Patrick Merlot, T. Kjærgaard, T. Helgaker, R. Lindh, F. Aquilante, S. Reine, and T. B. Pedersen. Attractive electron-electron interactions within robust local fitting approximations. J. Comput. Chem., 34:1486, 2013.

[17] David S. Hollman, Henry F. Schaefer, and Edward F. Valeev. Semi-exact concentric atomic density fitting: reduced cost and increased accuracy compared to standard density fitting. J. Chem. Phys., 140:064109, 2014.

[18] Florian Weigend and Reinhart Ahlrichs. Balanced basis sets of split valence, triple zeta valence and quadruple zeta valence quality for H to Rn: Design and assessment of accuracy. Phys. Chem. Chem. Phys., 7(18):3297-3305, 2005.

[19] F Weigend. Hartree-Fock exchange fitting basis sets for H to Rn. J. Comp. Chem., 29:167-175, 2008.

[20] Samuel F Manzer, Evgeny Epifanovsky, and Martin Head-Gordon. Efficient Implementation of the Pair Atomic Resolution of the Identity Approximation for Exact Exchange for Hybrid and Range-Separated Density Functionals. J. Chem. Theory Comput., 15(2):518-527, 2015.

[21] Samuel Manzer, Paul R. Horn, Narbe Mardirossian, and Martin Head-Gordon. Fast, accurate evaluation of exact exchange: The occ-RI-K algorithm. J. Chem. Phys., 143:024113, 2015.

[22] Frederick R. Manby. Density fitting in second-order linear-r12 Møller-Plesset perturbation theory. J. Chem. Phys., 119 (9):4607-4613, 2003.

[23] M. Feyereisen and G. Fitzgerald. Use of approximate integrals in ab initio theory. An application in MP2 energy calculations. Chem. Phys. Lett., 208:359-363, 1993.

[24] Hans-Joachim Werner, Frederick R. Manby, and Peter J. Knowles. Fast linear scaling second-order Møller-Plesset perturbation theory (MP2) using local and density fitting approximations. J. Chem. Phys., 118:8149-8160, 2003.

[25] Hans-Joachim Werner and Frederick R. Manby. Explicitly correlated second-order perturbation theory using density fitting and local approximations. J. Chem. Phys., 124:054114, 2006.

[26] Henk Eshuis, Julian Yarkony, and Filipp Furche. Fast computation of molecular random phase approximation correlation energies using resolution of the identity and imaginary frequency integration. J. Chem. Phys., 132:234114, 2010.

[27] Xinguo Ren, Patrick Rinke, Volker Blum, Jürgen Wieferink, Alexandre Tkatchenko, Andrea Sanfilippo, Karsten Reuter, and Matthias Scheffler. Resolution-of-identity approach to Hartree-Fock, hybrid density functionals, RPA, MP2 and GW with numeric atom-centered orbital basis functions. New J. Phys., 14:053020, 2012.

[28] Nelson H. F. Beebe and Jan Linderberg. Simplifications in the generation and transformation of two-electron integrals in molecular calculations. Int. J. Quantum Chem., 12:683-705, 1977.

[29] Henrik Koch, Alfredo Sánchez de Merás, and Thomas Bondo Pedersen. Reduced scaling in electronic structure calculations using Cholesky decompositions. J. Chem. Phys., 118:9481-9484, 2003.

[30] Francesco Aquilante, Thomas Bondo Pedersen, and Roland Lindh. Low-cost evaluation of the exchange fock matrix from cholesky and density fitting representations of the electron repulsion integrals. J. Chem. Phys., 126(19):194106, 2007.

[31] Thomas Bondo Pedersen, Francesco Aquilante, and Roland Lindh. Density fitting with auxiliary basis sets from cholesky decompositions. Theor. Chem. Acc., 124:1-10, 2009.

[32] Francesco Aquilante, Linus Boman, Jonas Boström, Henrik Koch, Roland Lindh, Alfredo Sánchez de Merás, and Thomas Bondo Pedersen. Cholesky Decomposition Techniques in Electronic Structure Theory. In Robert Zalesny, Manthos G. Papadopoulos, Paul G. Mezey, and Jerzy Leszczynski, editors, Linear-Scaling Techniques in Computational Chemistry and Physics. Methods and Applications, volume 13 of Challenges and Advances in Computational Chemistry and Physics, chapter 13, pages 301-343. Springer, 2011.

[33] G. R. Ahmadi and J. Almlöf. The coulomb operator in a gaussian product basis. Chem. Phys. Lett., 246:364, 1995. 
[34] Christopher A. White and Martin Head-Gordon. A j matrix engine for density functional theory calculations. J. Chem. Phys., 104:2620, 1996.

[35] Yihan Shao and Martin Head-Gordon. An improved j matrix engine for density functional theory calculations. Chem. Phys. Lett., 323(5):425 - 433, 2000.

[36] Manuel Guidon, Jürg Hutter, and Joost VandeVondele. Auxiliary Density Matrix Methods for Hartree-Fock Exchange Calculations. J. Chem. Theory Comput., 6:2348-2364, 2010.

[37] Patrick Merlot, Róbert Izsák, Alex Borgoo, Thomas Kjærgaard, Trygve Helgaker, and Simen Reine. Charge-constrained auxiliary-density-matrix methods for the hartree-fock exchange contribution. J. Chem. Phys., 141(9):094104, 2014.

[38] Frank Neese, Frank Wennmohs, Andreas Hansen, and Ute Becker. Efficient, approximate and parallel Hartree-Fock and hybrid DFT calculations. A 'chain-of-spheres' algorithm for the Hartree-Fock exchange. Chem. Phys., 356:98-1009, 2009.

[39] R. A. Friesner. Electronic ground state of iron(II)porphyrin. Ab initio SCF and Cl calculations and computed electron deformation densities. Chem. Phys. Lett., 116:39, 1985.

[40] Richard A. Friesner. Solution of the Hartree-Fock equations by a pseudospectral method: Application to diatomic molecules. J. Chem. Phys., 85(3):1462-1468, 1986.

[41] R. A. Friesner. Solution of the Hartree-Fock equations for polyatomic molecules by a pseudospectral method. J. Chem. Phys., 86:3522, 1987.

[42] J Olsen and P Jørgensen. Solution of the Hartree-Fock equations for polyatomic molecules by a pseudospectral method. J. Chem. Phys., 82:3235, 1985.

[43] P. Sałek, O. Vahtras, T. Helgaker, and H. Ågren. Density-functional theory of linear and nonlinear time-dependent molecular properties. J. Chem. Phys., 117:9630, 2002.

[44] J. Kussmann and C. Ochsenfeld. A density matrix-based method for the linear-scaling calculation of dynamic secondand third-order properties at the Hartree-Fock and Kohn-Sham density functional theory levels. J. Chem. Phys., 127: 204103, 2007.

[45] Andreas J. Thorvaldsen, Kenneth Ruud, Kasper Kristensen, Poul Jørgensen, and Sonia Coriani. A density matrixbased quasienergy formulation of the kohn-sham density functional response theory using perturbation- and timedependent basis sets. J. Chem. Phys., 129(21):214108, 2008.

[46] K. Sasagane, F. Aiga, and R. Itoh. Higher-order response theory based on the quasienergy derivatives: The derivation of the frequency-dependent polarizabilities and hyperpolarizabilities. J. Chem. Phys., 99:3738, 1993.

[47] O. Christiansen, C. Hättig, and P. Jørgensen. Response functions from Fourier component variational perturbation theory applied to a time-averaged quasienergy. Int. J. Quantum Chem., 68:1, 1998.

[48] H. Larsen, P. Jørgensen, J. Olsen, and T. Helgaker. Hartree-Fock and Kohn-Sham atomic-orbital based time-dependent response theory. J. Chem. Phys., 113:8908, 2000.

[49] S. Coriani, S. Høst, B. Jansík, L. Thøgersen, J. Olsen, P. Jørgensen, S. Reine, F. Pawłowski, T. Helgaker, and P. Sałek. A linear scaling implementation of molecular response theory in self-consistent field electronic-structure theory. J. Chem. Phys., 126:154108, 2007.

[50] Thomas Kjærgaard, Poul Jørgensen, Jeppe Olsen, Sonia Coriani, and Trygve Helgaker. Hartree-Fock and Kohn-Sham time-dependent response theory in a second-quantization atomic-orbital formalism suitable for linear scaling. J. Chem. Phys., 129:054106, 2008.

[51] D. N. Zubarev. Nonequilibrium Statistical Mechanics. Consultants Bureau, New York, 1974. 
[52] Magnus Ringholm, Dan Jonsson, and Kenneth Ruud. A general, recursive, and open-ended response code. J. Comput. Chem., 35(8):622-633, 2014.

[53] Daniel H. Friese, Maarten T. P. Beerepoot, Magnus Ringholm, and Kenneth Ruud. Open-ended recursive approach for the calculation of multiphoton absorption matrix elements. J. Chem. Theory Comput., 11(3):1129-1144, 2015.

[54] Daniel H. Friese, Magnus Ringholm, Bin Gao, and Kenneth Ruud. Open-ended recursive calculation of single residues of response functions for perturbation-dependent basis sets. J. Chem. Theory Comput., 11(10):4814-4824, 2015.

[55] Arnfinn Hykkerud Steindal, Maarten T. P. Beerepoot, Magnus Ringholm, Nanna Holmgaard List, Kenneth Ruud, Jacob Kongsted, and Jogvan Magnus Haugaard Olsen. Open-ended response theory with polarizable embedding: multiphoton absorption in biomolecular systems. Phys. Chem. Chem. Phys., 18:28339-28352, 2016.

[56] Christof Hättig and Florian Weigend. CC2 excitation energy calculations on large molecules using the resolution of the identity approximation. J. Chem. Phys., 113(13):5154-5161, 2000.

[57] Christof Hättig and Andreas Köhn. Transition moments and excited-state first-order properties in the coupled-cluster model CC2 using the resolution-of-the-identity approximation. J. Chem. Phys., 117(15):6939-6951, 2002.

[58] Christof Hättig and Kasper Hald. Implementation of RI-CC2 triplet excitation energies with an application to transazobenzene. Phys. Chem. Chem. Phys., 4:2111-2118, 2002.

[59] Christof Hättig. Geometry optimizations with the coupled-cluster model CC2 using the resolution-of-the-identity approximation. J. Chem. Phys., 118:7751-7761, 2003.

[60] Andreas Köhn and Christof Hättig. Analytic gradients for excited states in the coupled-cluster model CC2 employing the resolution-of-the-identity approximation. J. Chem. Phys., 119(10):5021-5036, 2003.

[61] Daniel H. Friese, Christof Hättig, and Kenneth Ruud. Calculation of two-photon absorption strengths with the approximate coupled cluster singles and doubles model cc2 using the resolution-of-identity approximation. Phys. Chem. Chem. Phys., 14:1175-1184, 2012.

[62] Daniel H. Friese, Nina O. C. Winter, Patrick Balzerowski, Raffael Schwan, and Christof Hättig. Large scale polarizability calculations using the approximate coupled cluster model CC2 and MP2 combined with the resolution-of-the-identity approximation. J. Chem. Phys., 136(17):174106, 2012.

[63] Daniel H. Friese, Christof Hättig, and Jörg Kussmann. Analytic molecular hessian calculations for cc2 and mp2 combined with the resolution of identity approximation. J. Chem. Theory Comput., 9(3):1469-1480, 2013.

[64] Daniel H. Friese and Christof Hättig. Optical rotation calculations on large molecules using the approximate coupled cluster model cc2 and the resolution-of-the-identity approximation. Phys. Chem. Chem. Phys., 16:5942-5951, 2014.

[65] Nora K. Graf, Daniel H. Friese, Nina O. C. Winter, and Christof Hättig. Excited state polarizabilities for CC2 using the resolution-of-the-identity approximation. J. Chem. Phys., 143(24):244108, 2015.

[66] Peter DegImann, Klaus May, Filipp Furche, and Reinhart Ahlrichs. Nuclear second analytical derivative calculations using auxiliary basis set expansions. Chem. Phys. Lett., 384:103-107, 2004.

[67] Rüdiger Bauernschmitt, Marco Häser, Oliver Treutler, and Reinhart Ahlrichs. Calculation of excitation energies within time-dependent density functional theory using auxiliary basis set expansions. Chem. Phys. Lett., 264:573-578, 1997.

[68] Frank Neese and Gottfried Olbrich. Efficient use of the resolution of the identity approximation in time-dependent density functional calculations with hybrid density functionals. Chem. Phys. Lett., 362(1-2):170 - 178, 2002.

[69] Stefan Grimme, Filipp Furche, and Reinhart Ahlrichs. An improved method for density functional calculations of the frequency-dependent optical rotation. Chem. Phys. Lett., 361(3-4):321 - 328, 2002. 
[70] Filipp Furche and Dmitrij Rappoport. $\{$ III $\}$ - density functional methods for excited states: Equilibrium structure and electronic spectra. In M. Olivucci, editor, Computational Photochemistry, volume 16 of Theoretical and Computational Chemistry, pages 93 - 128. Elsevier, 2005.

[71] Dmitrij Rappoport and Filipp Furche. Analytical time-dependent density functional derivative methods within the RI-J approximation, an approach to excited states of large molecules. J. Chem. Phys., 122(6):064105, 2005.

[72] Chandan Kumar, Thomas Kjærgaard, Trygve Helgaker, and Heike Fliegl. Nuclei-selected atomic-orbital responsetheory formulation for the calculation of NMR shielding tensors using density-fitting. J. Chem. Phys., 145(23):234108, 2016.

[73] Stefan Loibl, Frederick R. Manby, and Martin Schütz. Density fitted, local hartree-fock treatment of nmr chemical shifts using london atomic orbitals. Molecular Physics, 108(3-4):477-485, 2010.

[74] Carl-Mikael Suomivuori, Nina O. C. Winter, Christof Hättig, Dage Sundholm, and Ville R. I. Kaila. Exploring the lightcapturing properties of photosynthetic chlorophyll clusters using large-scale correlated calculations. Journal of Chemical Theory and Computation, 12(6):2644-2651, 2016.

[75] F. London. Théorie quantique des courants interatomiques dans les combinaisons aromatiques. J. Phys. Radium, 8:397409, 1937.

[76] L. J. Sham and W. Kohn. Self-consistent equations including exchange and correlation effects. Phys. Rev. A, 140:1133, 1965.

[77] M.A.L. Marques and E.K.U. Gross. Time-dependent Density Functional Theory. Annu. Rev. Phys. Chem., 55(1):427-455, 2004.

[78] Kieron Burke, Jan Werschnik, and E. K. U. Gross. Time-dependent density functional theory: Past, present, and future. J. Chem. Phys., 123:062206, 2005.

[79] Klaus Capelle. A bird's-eye view of density-functional theory. Brazilian Journal of Physics, 36:1318 - $1343,2006$.

[80] M.E. Casida and M. Huix-Rotllant. Progress in Time-Dependent Density-Functional Theory. Annu. Rev. Phys. Chem., 63 (1):287-323, 2012.

[81] T. Helgaker, P. Jørgensen, and J. Olsen. Molecular Electronic-Structure Theory. Wiley, Chichester, 2000.

[82] T Helgaker, H Larsen, J Olsen, and P Jørgensen. Direct optimization of the AO density matrix in Hartree-Fock and Kohn-Sham theories. Chem. Phys. Lett., 327:397-403, 2000.

[83] H Larsen, P Jørgensen, J Olsen, and T Helgaker. Hartree-Fock and Kohn-Sham atomic-orbital based time-dependent response theory. J. Chem. Phys., 113:8908-8917, 2000.

[84] Linus Boman, Henrik Koch, and Alfredo Sánchez de Merás. Method specific Cholesky decomposition: Coulomb and exchange energies. J. Chem. Phys., 129:134107, 2008.

[85] Kestutis Aidas, Celestino Angeli, Keld L. Bak, Vebjørn Bakken, Radovan Bast, Linus Boman, Ove Christiansen, Renzo Cimiraglia, Sonia Coriani, Pål Dahle, Erik K. Dalskov, Ulf Ekström, Thomas Enevoldsen, Janus J. Eriksen, Patrick Ettenhuber, Berta Fernández, Lara Ferrighi, Heike Fliegl, Luca Frediani, Kasper Hald, Asger Halkier, Christof Hättig, Hanne Heiberg, Trygve Helgaker, Alf Christian Hennum, Hinne Hettema, Eirik Hjertenæs, Stinne Høst, Ida-Marie Høyvik, Maria Francesca lozzi, Branislav Jansík, Hans Jørgen Aa. Jensen, Dan Jonsson, Poul Jørgensen, Joanna Kauczor, Sheela Kirpekar, Thomas Kjærgaard, Wim Klopper, Stefan Knecht, Rika Kobayashi, Henrik Koch, Jacob Kongsted, Andreas Krapp, Kasper Kristensen, Andrea Ligabue, Ola B. Lutnæs, Juan I. Melo, Kurt V. Mikkelsen, Rolf H. Myhre, Christian Neiss, Christian B. Nielsen, Patrick Norman, Jeppe Olsen, Jógvan Magnus H. Olsen, Anders Osted, Martin J. Packer, Filip Pawlowski, Thomas B. Pedersen, Patricio F. Provasi, Simen Reine, Zilvinas Rinkevicius, Torgeir A. Ruden, Kenneth Ruud, Vladimir V. Rybkin, Pawel Sałek, Claire C. M. Samson, Alfredo Sánchez de Merás, Trond Saue, Stephan P. A. Sauer, 
Bernd Schimmelpfennig, Kristian Sneskov, Arnfinn H. Steindal, Kristian O. Sylvester-Hvid, Peter R. Taylor, Andrew M. Teale, Erik I. Tellgren, David P. Tew, Andreas J. Thorvaldsen, Lea Thøgersen, Olav Vahtras, Mark A. Watson, David J. D. Wilson, Marcin Ziolkowski, and Hans Ågren. The Dalton quantum chemistry program system. WIREs Comput Mol Sci, 4 (3):269-284, 2014.

[86] LSDalton, a linear scaling molecular electronic structure program, Release Dalton2015.1, 2015. http://daltonprogram.org. .

[87] Marko Schreiber, Mario R. Silva-Junior, Stephan P. A. Sauer, and Walter Thiel. Benchmarks for electronically excited states: CASPT2, CC2, CCSD, and CC3. J. Chem. Phys., 128(13):134110, 2008.

[88] C. Ochsenfeld, C. A. White, and M. Head-Gordon. Linear and sublinear scaling formation of Hartree-Fock-type exchange matrices. J. Chem. Phys., 109:1663-1669, 1998.

[89] Frank Jensen. Unifying General and Segmented Contracted Basis Sets. Segmented Polarization Consistent Basis Sets. Journal of Chemical Theory and Computation, 10(3):1074-1085, 2014.

[90] Florian Weigend. Hartree-Fock exchange fitting basis sets for H to Rn. J. Comput. Chem., 29(2):167-175, 2008.

[91] Simen Reine, Erik Tellgren, Trygve Helgaker, Ulf Ekström, Robert Izsak, Frank Jensen, and Thomas Kjærgaard. Basis sets for the Auxiliary-Density-Matrix Method. in preparation, 2017.

[92] Optimized ADMM basis sets used in the present work can be downloaded upon request on: //gitlab.com/chandankumar/ADMM-basis-sets-ijqc.

[93] M. J. G. Peach, P. Benfield, T. Helgaker, and D. J. Tozer. Excitation energies in density functional theory: An evaluation and a diagnostic test. J. Chem. Phys., 128:044118, 2008.

[94] Aron J. Cohen, Paula Mori-Aánchesz, and Weitao Yang. Challenges for Density Functional Theory. Chem. Rev., 112(1): 289-320, 2012.

[95] George Maroulis. Static hyperpolarizability of the water dimer and the interaction hyperpolarizability of two water molecules. J. Chem. Phys., 113(5):1813-1820, 2000.

[96] Ihsan M. Kenawia, Aladin H. Kamelb, and Rifaat H. Hilala. BSSE effects on the static dipole polarizability and first dipole hyperpolarizability of diclofenac sodium. J. Mol. Struct. (Theochem), 851(1-3):46-53, 2008.

[97] J. Schirmer. Beyond the random-phase approximation: A new approximation scheme for the polarization propagator. Phys. Rev. A, 26:2395-2416, 1982.

[98] A. B. Trofimov and J. Schirmer. An efficient polarization propagator approach to valence electron excitation spectra. Phys. Rev. B, 28:2299-2324, 1995.

[99] Godfrey S. Beddard. Exciton coupling in the photosystem i reaction center. The Journal of Physical Chemistry B, 102(52): 10966-10973, 1998.

[100] Julian Adolphs, Frank Müh, Mohamed El-Amine Madjet, Marcel Schmidt am Busch, and Thomas Renger. Structurebased calculations of optical spectra of photosystem i suggest an asymmetric light-harvesting process. Journal of the American Chemical Society, 132(10):3331-3343, 2010.

[101] Eberhard Schlodder, Friedhelm Lendzian, Jenny Meyer, Marianne Çetin, Marc Brecht, Thomas Renger, and Navasard V. Karapetyan. Long-wavelength limit of photochemical energy conversion in photosystem i. Journal of the American Chemical Society, 136(10):3904-3918, 2014.

[102] Eberhard Schlodder, Vladimir V. Shubin, Eithar El-Mohsnawy, Matthias Roegner, and Navassard V. Karapetyan. Steadystate and transient polarized absorption spectroscopy of photosytem i complexes from the cyanobacteria arthrospira platensis and thermosynechococcus elongatus. Biochimica et Biophysica Acta (BBA) - Bioenergetics, 1767:732 - 741, 2007. ISSN 0005-2728. Structure and Function of Photosystems. 
[103] A. D. Becke. Density-fnnctional exchange-energy approximation with correct asymptotic behavior. Phys. Rev. A, 38: 3098, 1988.

[104] C. Lee, W. Yang, and R. G. Parr. Development of the colic-salvetti correlation-energy formula into a functional of the electron density. Phys. Rev. B, 37:785, 1988.

[105] A D Becke. Density-functional thermochemistry. III. The role of exact exchange. J. Chem. Phys., 98:5648-5652, 1993.

[106] Takeshi Yanai, David P Tew, and Nicholas C Handy. A new hybrid exchange-correlation functional using the Coulombattenuating method (CAM-B3LYP). Chem. Phys. Lett., 393(1-3):51 - 57, 2004.

[107] P. A. M. Dirac. Note on exchange phenomena in the thomas atom. Proc. Camb. Phil. Soc., 26:376, 1930.

[108] Hisayoshi likura, Takao Tsuneda, Takeshi Yanai, and Kimihiko Hirao. A long-range correction scheme for generalizedgradient-approximation exchange functionals. J. Chem. Phys., 115:3540-3544, 2001.

[109] S. J. Vosko, L. Wilk, and M. Nusair. Accurate spin-dependent electron liquid correlation energies for local spin density calculations: a critical analysis. Can. J. Phys., 58:1200, 1980. 